Review Article

\title{
Mitochondrial Dysfunction in Chronic Respiratory Diseases: Implications for the Pathogenesis and Potential Therapeutics
}

\author{
Wen-cheng Zhou $\mathbb{D}^{1},{ }^{1}$ Jiao Qu $\mathbb{D}^{2},{ }^{2}$ Sheng-yang Xie $\mathbb{D}^{1},{ }^{1}$ Yang Sun $\mathbb{D}^{2},{ }^{2}$ and Hong-wei Yao $\mathbb{D}^{3}$ \\ ${ }^{1}$ Department of Pharmacy, The First Affiliated Hospital of Zhejiang Chinese Medical University (Zhejiang Provincial Hospital of \\ Traditional Chinese Medicine), Hangzhou 310006, China \\ ${ }^{2}$ State Key Laboratory of Pharmaceutical Biotechnology, Department of Biotechnology and Pharmaceutical Sciences, School of \\ Life Science, Nanjing University, Nanjing 210023, China \\ ${ }^{3}$ School of Pharmacy, Anhui Medical University, Hefei 230032, China
}

Correspondence should be addressed to Yang Sun; yangsun@nju.edu.cn and Hong-wei Yao; yhgwei@hotmail.com

Received 7 May 2021; Revised 30 June 2021; Accepted 16 July 2021; Published 27 July 2021

Academic Editor: Stephan Immenschuh

Copyright (c) 2021 Wen-cheng Zhou et al. This is an open access article distributed under the Creative Commons Attribution License, which permits unrestricted use, distribution, and reproduction in any medium, provided the original work is properly cited.

\begin{abstract}
Mitochondria are indispensable for energy metabolism and cell signaling. Mitochondrial homeostasis is sustained with stabilization of mitochondrial membrane potential, balance of mitochondrial calcium, integrity of mitochondrial DNA, and timely clearance of damaged mitochondria via mitophagy. Mitochondrial dysfunction is featured by increased generation of mitochondrial reactive oxygen species, reduced mitochondrial membrane potential, mitochondrial calcium imbalance, mitochondrial DNA damage, and abnormal mitophagy. Accumulating evidence indicates that mitochondrial dysregulation causes oxidative stress, inflammasome activation, apoptosis, senescence, and metabolic reprogramming. All these cellular processes participate in the pathogenesis and progression of chronic respiratory diseases, including chronic obstructive pulmonary disease, pulmonary fibrosis, and asthma. In this review, we provide a comprehensive and updated overview of the impact of mitochondrial dysfunction on cellular processes involved in the development of these respiratory diseases. This not only implicates mechanisms of mitochondrial dysfunction for the pathogenesis of chronic lung diseases but also provides potential therapeutic approaches for these diseases by targeting dysfunctional mitochondria.
\end{abstract}

\section{Introduction}

Mitochondria are subcellular organelles that originate from a bacterial symbiont, containing two separate and functionally distinct outer and inner membranes that separate the intermembrane space and matrix compartment $[1,2]$. It is considered the powerhouse of cells with energy generation in the forms of adenosine $5^{\prime}$-triphosphate (ATP) via oxidative phosphorylation (OXPHOS) on the electron transport chain (ETC) [3]. Mitochondria differ from other organelles and have their own maternally inherited DNA (mtDNA) [4]. mtDNA contains 37 genes coded for two ribosomal RNAs, 22 transfer RNAs, and the remainder 13 encoding proteins involved in OXPHOS. The mechanism of mtDNA replication is complex, which involves several critical enzymes, including the heterodimer mtDNA polymerase, DNA heli- case Twinkle, mitochondrial RNA polymerase, mitochondrial single-strand DNA-binding protein, topoisomerase 3, and DNA ligase III [5-7]. Existing studies reveal that mtDNA replication is critically important to mitochondrial biogenesis [8]. The latter is a process of growth and division of preexisting mitochondria rather than de novo synthesis, depending on the coordination of both mitochondrial and nuclear genomes [9]. Mitochondrial numbers are regulated by mitochondrial biogenesis to meet the energy demands of the cell, which process is associated with mitochondrial fusion and fission. Fission causes additional smaller mitochondria, which promotes mitophagy, cell proliferation, and reactive oxygen species (ROS) production. Fusion results in the interconnection of the mitochondrial network as well as communication with endoplasmic reticulum (ER) and diffusion of matrix content among mitochondria, which 
dilutes accumulated mtDNA mutations and oxidized proteins $[9,10]$. At the molecular levels, both optic atrophy 1 at the inner membrane and mitofusin 1 and 2 at the outer membrane mediate mitochondrial fusion. Fission is controlled by dynamin-related protein 1 and its docking proteins, mitochondrial fission protein 1, mitochondrial fission factor, and mitochondrial elongation factors 1 and 2 [11, 12]. In addition, mitophagy is an important control system for the selective elimination of damaged mitochondria. Furthermore, the close apposition between the ER and mitochondria is conducive to intimately communicate, forming very dynamic platforms termed mitochondria-associated membranes (MAM), which facilitate calcium flux into the mitochondria. Mitochondrial calcium flux participates in regulating essential functions, including metabolism, energy production, and apoptosis [13]. Hence, maintaining mitochondrial function and homeostasis is essential for healthy cellular function and survival. Mitochondrial homeostasis is associated with stabilization of mitochondrial membrane potential (MMP), balance of mitochondrial calcium, integrity of mtDNA, and timely clearance of damaged mitochondria via mitophagy (Figure 1). Once mitochondrial homeostasis is disrupted, it can disturb the OXPHOS process and induce accumulation and generation of side products, including ROS, which further cause mitochondrial dysfunction, forming a vicious cycle harmful to cell function [14].

Mitochondrial homeostasis can be disrupted by environmental insults including tobacco smoke, air pollutants, inflammation, infection, hypoxia, and hyperoxia, resulting in mitochondrial dysfunction. This is characterized by increased mtROS, reduced MMP, mtDNA damage and release, and abnormal calcium signaling and storage. These abnormalities are able to cause oxidative stress, inflammasome activation, apoptosis, senescence, and metabolic reprogramming [15] (Figure 2). The role of mitochondria in disease progression is extensively investigated and suggests a direct link between mitochondrial dysfunction and the development of chronic respiratory diseases. Mitochondrial dysfunction is considered an important pathogenic mechanism of chronic respiratory diseases, including chronic obstructive pulmonary disease (COPD), pulmonary fibrosis, and asthma $[2,16,17]$. In this review, we discuss how mitochondrial dysfunction results in abnormal cellular processes and its role in the development of these chronic respiratory diseases. This not only enhances understanding of mechanisms of mitochondrial dysfunction underlying the pathogenesis of chronic respiratory diseases but also suggests potential therapeutic approaches for these diseases by targeting dysregulated mitochondria.

\section{Mitochondrial Dysregulation in Pathological Processes}

2.1. Abnormal mtROS Generation. Mitochondria are the major source of cellular ROS during OXPHOS. Electrons that leak out of the complex I (NADH dehydrogenase) oxidize oxygen to generate superoxide anion $\left(\mathrm{O}_{2}{ }^{-}\right) \cdot \mathrm{O}_{2}{ }^{-}$can be dismutated into hydrogen peroxide $\left(\mathrm{H}_{2} \mathrm{O}_{2}\right)$ by manganese superoxide dismutase (MnSOD), a mitochondrion-specific antioxidant. Glutathione peroxidase converts $\mathrm{H}_{2} \mathrm{O}_{2}$ to water. Meanwhile, $\mathrm{H}_{2} \mathrm{O}_{2}$ can form the highly reactive hydroxyl radical $(\mathrm{OH})$ by reacting with metal ions [18]. Hence, production of ROS in the respiratory chain is dependent on the release of electrons out of the ETC followed by the formation of these free radicals. Although the ETC is a major ROS producer in mitochondria, there are still other mitochondrial proteins involved in mtROS generation. These proteins include enzymes in the tricarboxylic acid cycle (i.e., aconitase, pyruvate dehydrogenase, and $\alpha$-ketoglutarate dehydrogenase), inner mitochondrial membrane proteins (e.g., cytochrome P450 enzymes and glycerol-3-phosphate dehydrogenase), and outer mitochondrial membrane protein (e.g., cytochrome b5 reductase and monoamine oxidase) [19]. Under physiological conditions, low levels of mtROS serve as second messengers in coordinating biological and physiological processes [20, 21].

Abnormal mtROS has a causal role in mitochondrial dysfunction [22]. A variety of environmental toxins/oxidants and hyperoxia cause mtROS overproduction, which could damage mitochondrial protein, mitochondrial lipid membrane, and mtDNA [23]. Zorov et al. revealed that mtROS release causes the loss of MMP and increases mitochondrial permeability, called mitochondrial permeability transition (MPT) induction [24]. MPT induction alters the rigidity of the mitochondrial membrane and weakens protein-protein interactions which is necessary for the proper function of the respiratory chain [25]. Thus, the mtROS bursts are partly attributed to a block of the ETC caused by MPT induction [26]. Due to a lack of protective histones, mtDNA is susceptible to damage by mtROS, leading to mtDNA mutation. In turn, mtDNA mutation impairs the OXPHOS process further resulting in mtROS production [27-29]. These findings suggest vicious feedback between mtROS and mitochondrial dysfunction.

It has been shown that mtROS accumulation causes a range of cellular processes, including inflammation, apoptosis, and senescence [30]. For instance, Silvia et al. discovered ethanol-induced NOD-like receptor family pyrin domaincontaining-3 (NLRP3) inflammasome activation upon mtROS generation. A mitochondrion-targeted antioxidant Mito-TEMPO abrogates mtROS release and reduces the upregulation of IL-1 $\beta$ and IL-18 induced by ethanol, effectively inhibiting NLRP3 activation [31]. Similar results are observed in the kidneys of patients with diabetic nephropathy and $\mathrm{db} / \mathrm{db}$ mice; mtROS overproduction is accompanied by increases in NLRP3/IL- $1 \beta$ expression, which can be ameliorated by MitoQ, a mitochondria-targeted antioxidant [32]. In addition, mtROS increases mitochondrial membrane permeability. This leads to the escape of mtDNA into the cytoplasm, and mtDNA binds and activates the NLRP3 inflammasome [30, 33, 34]. Besides NLRP3 inflammasome, $\mathrm{mtROS}$ is able to activate a redox-sensitive mediator nuclear factor- $\kappa \mathrm{B}(\mathrm{NF}-\kappa \mathrm{B})$, which regulates the transcription of various proinflammatory cytokines $[35,36]$.

Growing evidence indicates that mtROS accumulation modulates cellular apoptosis. Cytochrome C (Cyt c) release from mitochondria is a key event in initiating apoptosis [37]. Increased production of mtROS causes mitochondrial 


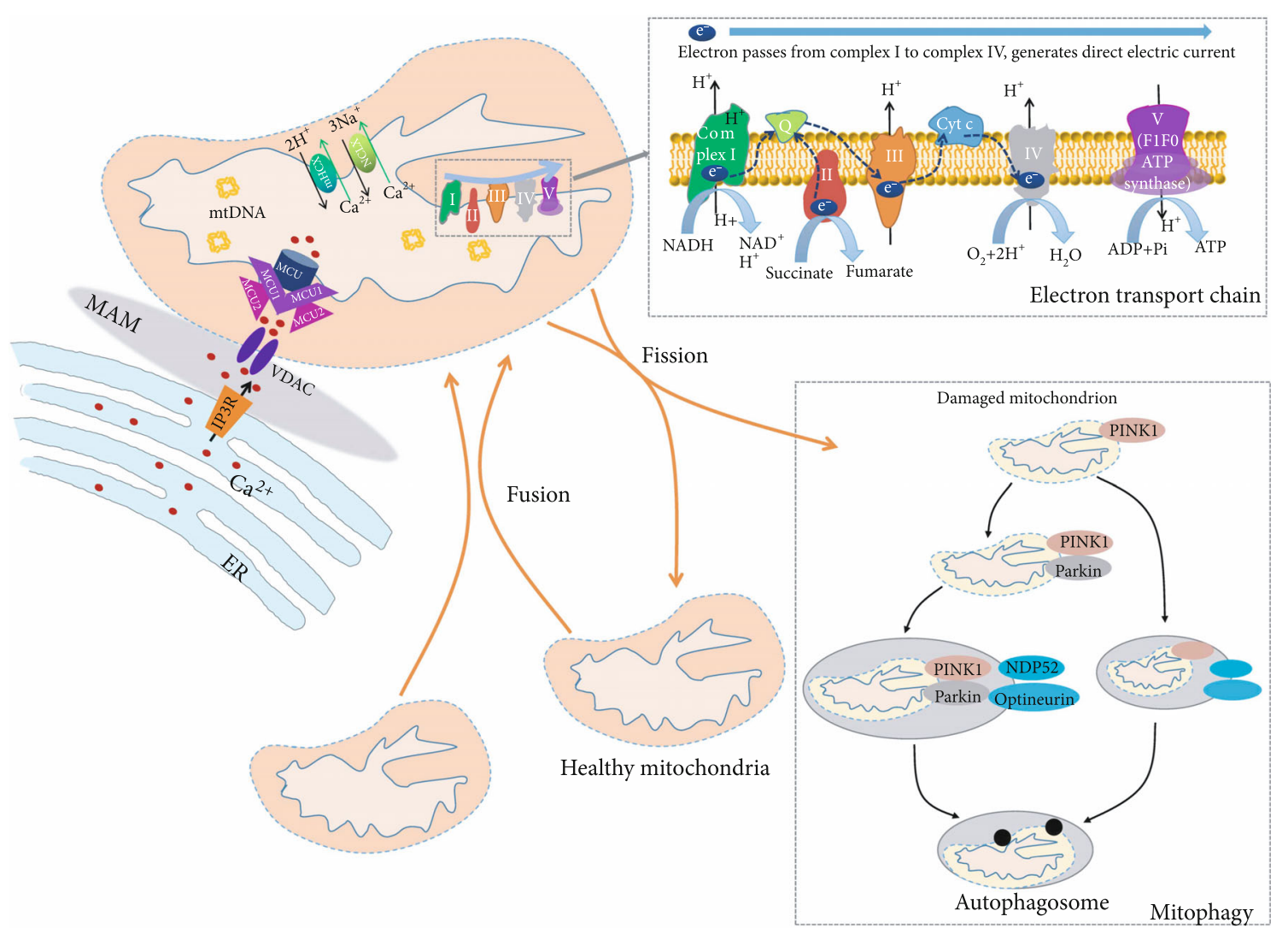

FIGURE 1: Normal mitochondria are sustained by stabilization of MMP, balance of mitochondrial calcium, integrity of mtDNA, and timely clearance of damaged mitochondria via mitophagy. The electron flows from complex I to complex IV, which creates a direct electric current that terminates in subunit II of complex IV for MMP. This is accompanied by the synthesis of ATP. Mitochondria are closely linked to the ER. They intimately and dynamically communicate, thereby forming very dynamic platforms termed MAM. The delivery of ER-mitochondria calcium mainly depends on IP3 receptors (IP3R) and voltage-dependent anion channel (VDAC). Mitochondrial calcium uptake is mediated by the MCU complex, while calcium release depends on MHCX and NCLX systems. Mitochondrion harbors its own DNA, which encodes many critical proteins for the assembly and activity of mitochondrial respiratory complexes. Mitochondria are dynamic through fission and fusion. Mitophagy is a selective and adaptive response and plays a critical role in surveilling mitochondrial quality and preventing accumulation of dysfunctional mitochondria.

dysfunction including membrane potential loss and ATP depletion, which promotes the release of mitochondrial Cyt c, and activates caspase-9 and caspase-3 [38]. A recent study showed that Cyt $\mathrm{c}$ release is positively associated with the generation of mtROS [39]. A separate study discovered that free fatty acids result in elevated mtROS generation, promoting mitochondrial accumulation of $\mathrm{p} 53$ followed by recruiting proapoptotic factor Bax, initiating apoptotic events. In contrast, treatment with MitoQ inhibits mtROS generation and alleviates apoptosis in high fat-fed mice [40]. Similarly, cudraflavone $\mathrm{C}$ induces apoptosis in melanoma cells by increasing mtROS production, enhances the phosphorylation of MAPKs (p38, ERK, and JNK), and increases the expression of apoptotic proteins (Bax, Apaf-1, Cyt c, caspase-9, and caspase-3/7). Pretreatment with Mito-TEMPO alleviates MAPK activation, expression of apoptotic proteins, and apoptosis [41].

A large body of studies have been published to support the role of mitochondrial oxidant production in the senescence/aging process, identifying that the production of mtROS is the major determinant of senescence/aging [42, 43]. Overexpression of catalase targeted to mitochondria pre- vents cardiac aging [44]. The accumulation of $m t R O S$ and mitochondrial homeostasis imbalance trigger p53/p21 and/or $\mathrm{p} 16 / \mathrm{pRb}$ pathways, leading to cellular senescence [45-47]. Indeed, mitochondrial dysfunction is considered an inducer of cellular senescence where mtROS is the most studied factor through persistent mtDNA damage. Due to the lack of histone, limited DNA repair capabilities, and proximity of mtDNA to the site of mtROS generation, mtDNA is sensitive to mtROS stimuli [48]. Excessive mtROS induces various types of mtDNA damage, including mtDNA mutation and decreased mtDNA copy number, which is thought to have a causal role in many age-related pathologies $[27,49]$. In addition, excessive mtROS triggers the activation of the mitochondrial permeability transition pore (PTP). This further increases production and release of mtROS, which damages both mitochondrial and nuclear DNA, phospholipids, and proteins. PTP induction also releases matrix NAD that is hydrolyzed in the intermembrane space, thereby resulting in the depletion of cellular NAD, and this accelerates senescence $[50,51]$. These results indicate that mtROS-induced cellular senescence mainly depends on mitochondrial oxidative damage, such as mtDNA mutations 


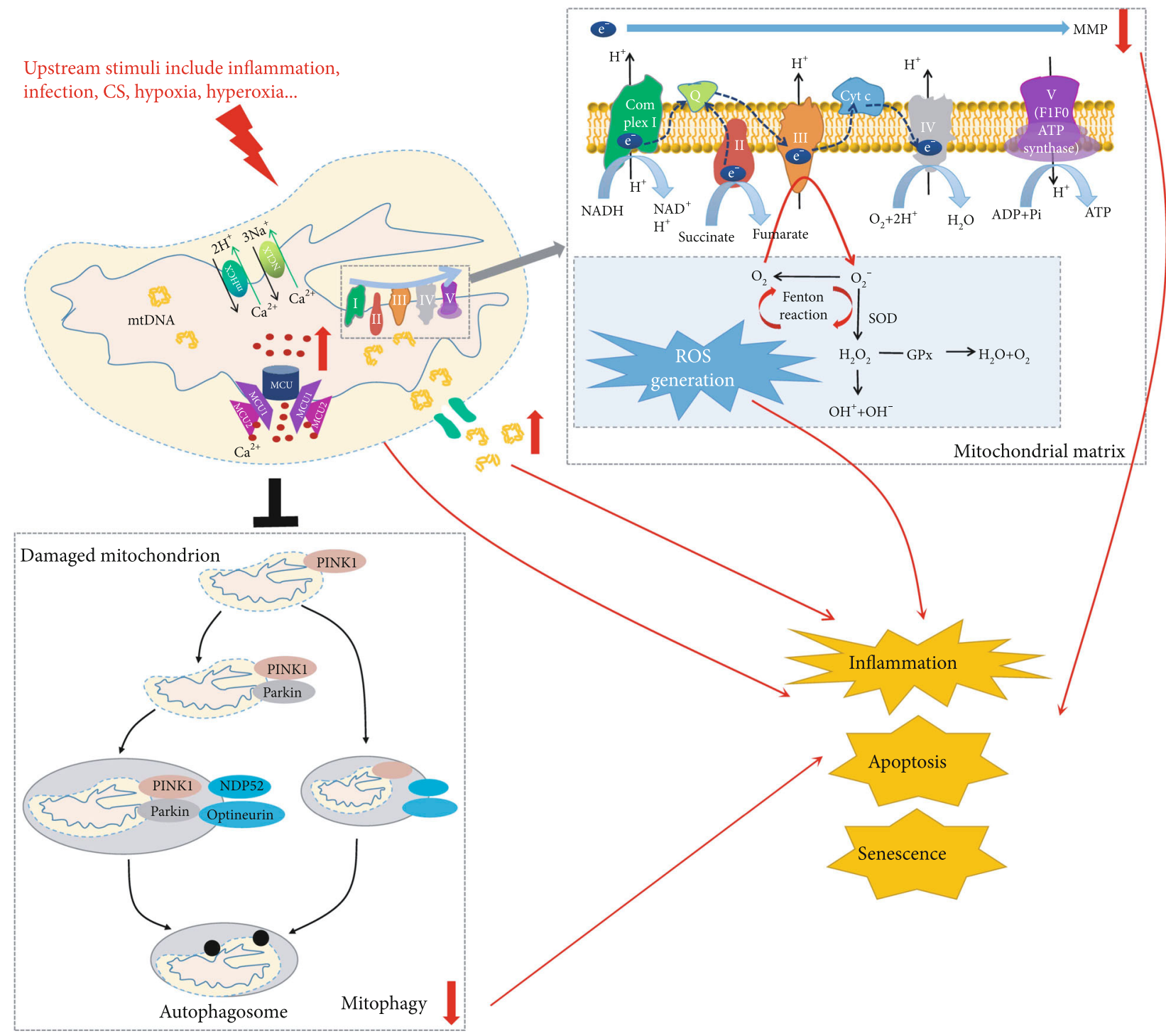

FIGURE 2: Mitochondrial dysfunction is characterized by increased mtROS, reduced MMP, increased calcium influx, mtDNA damage and release, and abnormal mitophagy. Mitochondrial homeostasis can be disrupted by upstream stimuli including inflammation, infection, CS, hypoxia, and hyperoxia, resulting in mitochondrial dysfunction. mtROS production in the respiratory chain depends on the release of electrons out of the ETC. Electrons that leak out of the ETC react with oxygen to produce $\mathrm{O}^{2-}$, which detoxifies into $\mathrm{H}_{2} \mathrm{O}_{2}$ via SOD. Glutathione peroxidase $(\mathrm{GPx})$ converts $\mathrm{H}_{2} \mathrm{O}_{2}$ to water. If unquenched, $\mathrm{H}_{2} \mathrm{O}_{2}$ can form the highly reactive $\mathrm{OH}$ by reacting with metal ions. Reduced MMP and mitochondrial calcium overload are important indicators of mitochondrial dysfunction, which has a profound influence on oxidative stress, inflammatory response, and apoptosis. mtDNA mutation and release impair mitochondrial respiratory chain, amplify mtROS, and accelerate mitochondrial dysfunction. Defective mitophagy results in the accumulation of damaged mitochondria. These abnormalities are able to cause oxidative stress, inflammasome activation, apoptosis, and senescence.

and mitochondrial membrane permeability. However, it is also possible that mtROS serves as signaling molecules to induce cellular senescence, independent of mitochondrial oxidative damage.

2.2. Reduced MMP. The MMP depends on ETC or the F1F0 ATP synthase system. It is an essential component in the process of energy storage during OXPHOS [52]. Under normal conditions, the ETC is the basic MMP producing system; the electron passes from complex I to complex IV and then generates MMP [53]. When the ETC function is abnormal, the F1F0 ATP synthase maintains MMP by reversing its action and hydrolyzing ATP. The ATP hydrolysis creates a direct electric current that is transferred from F1 to F0 where it is used to charge MMP [54]. MMP forms the transmembrane potential of hydrogen ions, which is harnessed to generate ATP [52]. Stabilization of MMP is the key determinant of mitochondrial performance, mitochondrial permeability, mitochondrial viability, nutrient import/output, and other important cellular functions [54].

Healthy mitochondria maintain high membrane potential, whereas the destruction of mitochondrial function can cause depolarization or a decrease in MMP [55]. Reduced MMP has been recognized as an important indicator of 
mitochondrial dysfunction and has a profound influence on inflammatory response and cellular apoptosis [56, 57]. Compared to controls, patients with systemic inflammatory response syndrome caused by sepsis, trauma, or others have a significant increase in platelet MMP depolarization [56]. Similar results are identified in contentious-flow left ventricular assist device patients with systemic inflammatory response syndrome; depolarization of MMP is significantly enhanced [58]. Consistently, age-related retinal inflammation is reduced by $670 \mathrm{~nm}$ light via increased MMP [59]. These results suggest that reduced MMP is associated with inflammation activation. Moreover, mitochondrial depolarization triggers inflammation, dependent on mitochondrial membrane permeability opening, which allows the release of mitochondrial components mtROS, mtDNA, or intermembrane space proteins into the cytosol [60]. MMP reduction-triggered inflammation activation may be more intricate, which still needs to be investigated.

The decrease in MMP can be triggered by the withdrawal of growth factors, deficiency of the extracellular glucose supply, blockage of respiration, or uncoupling of the inner membrane. Reduced MMP is a critical event in inducing cellular apoptosis $[61,62]$. There is an evidence linking the decline in MMP to structural changes of mitochondria, including matrix condensation and cristal unraveling. These structural changes result in Cyt c release from the cristae to the intermembrane space and cytosol [57]. The research further discovers that when mitochondria are depolarized, matrix volume decreases and density increases, but when the mitochondria are energized, it is the opposite. This suggests that manipulation of mitochondrial depolarization alters mitochondrial configuration, thereby influencing apoptosis. Mitochondrial membrane depolarization could sensitize human granulosa tumor cells to induction of apoptosis [63]. In addition, when mitochondria are incubated at pH6.4, oxidizable substrate-deprived, condensed mitochondria undergo decondensation of their matrix, as compared to incubation at physiological pH 7.4 [57]. Hence, the manipulation of the proton gradient across the mitochondrial membrane by acidifying the medium may be efficient for repairing MMP and controlling apoptosis.

2.3. Mitochondrial Calcium Dysregulation. The ER lumen is identified as the major intracellular calcium storage compartment. Mitochondria are closely linked to the ER, and approximately $20 \%$ of the mitochondrial surface is found in close proximity to the ER [64]. The close apposition between the ER and mitochondria facilitates calcium flux into the mitochondria. The ER calcium release machinery mainly involves in the inositol 1,4,5-trisphosphate (IP3) receptor [65]. The transferring of calcium into the mitochondrial intermembrane space is mediated by a voltage-dependent anion channel on the mitochondrial outer membrane at the ERmitochondria contacts [13]. Calcium enters into the mitochondrial matrix as a key intracellular second messenger by binding and triggering changes in protein shape and charge, regulating proteins, enzymes, and transporters responsible for ATP synthesis [66]. Calcium has been shown to regulate the TCA cycle by activating three mitochondrial dehydroge- nases, such as pyruvate dehydrogenase, isocitrate dehydrogenase, and $\alpha$-ketoglutarate dehydrogenase, thereby controlling the rate of ATP synthesis [67]. More recently, some reports demonstrate that calcium could directly stimulate ATP production through activation of complex $\mathrm{V}$ and the F1F0 ATP synthase and promote electron transport via complex III of ETC $[68,69]$. Mitochondrial calcium also modulates other cell functions, including MPT pore opening and Cyt $\mathrm{c}$ release $[23,70,71]$.

Mitochondrial calcium balance is controlled by mitochondrial calcium uptake and release systems. There is a general consensus that mitochondrial calcium uptake is mediated by a macromolecular structure, the mitochondrial calcium uniporter (MCU) complex [72]. The MCU contains several components, including the MCU paralog MCUb, a family of related EF-hand-containing proteins (MICU1, MICU2, and MICU3), and EMRE, which composition appears to differ among various cell lines and tissues [73]. Calcium release depends on two different mechanisms, one mediated by a $2 \mathrm{H}^{+} / \mathrm{Ca}^{2+}$ antiporter (mHCX) expressed in most of the cells and the other by the control of the $\mathrm{Na}^{+} / \mathrm{Ca}^{2+}$ exchanger encoded by the NCLX gene $[74,75]$. In addition, mitochondrial calcium homeostasis is also associated with the ER calcium balance. Existing evidence proposes that mitochondrial calcium disorder is possibly a result of ER calcium imbalance. Sarcoplasmic/endoplasmic reticulum $\mathrm{Ca}^{2+}$-ATPase (SERCA) is a pump that transports calcium ions from the cytoplasm into the ER. SERCA attenuates calcium overload and prevents activation of xanthine oxidase, resulting in decreased mtROS and improved mitochondrial quality control [76].

Mitochondrial calcium overload is closely associated with the production of oxidative stress $[77,78]$. As shown by in vitro experiments, the addition of calcium to isolated rat heart mitochondria in the presence of antimycin A, a complex III inhibitor, induces ROS formation [79]. Inhibition of the activity of the MCU by an MCU inhibitor RU360 or small interfering RNA (siRNA) decreases mitochondrial calcium uptake, which renders the cells resistant to oxidative stress in in vitro experiments. In contrast, overexpression of the MCU causes higher sensitivity to the oxidative stress of cells $[78,80]$. Silencing of NCLX expression decreases mitochondrial calcium release and aggravates high glucoseinduced oxidative stress [81]. A recent study has shown that mitochondrial calcium overload triggers the net production of ROS through activation of mitochondrial PTP with the release of Cyt c, inhibition of respiratory chain, release of pyridine nucleotides, and loss of intramitochondrial glutathione necessary for the detoxification of peroxides [82].

NLRP3 inflammasome could be activated by calcium signaling [83]. Increased intracellular calcium concentration leads to calcium accumulation in the mitochondrial matrix via MCU leading to MMP loss, NLRP3 inflammasome activation, and IL- $1 \beta$ release [84]. Silencing of NCLX expression promotes NLRP3 inflammasome activation [81]. Rimessi et al. demonstrated that inhibition of MCU by the MCU inhibitor KB-R7943 could be beneficial for alleviating the $P$. aeruginosa-dependent inflammatory response in cystic fibrosis [85]. NLRP3 inflammasome activation depends on MCU, 
indicating that mitochondrial calcium is a novel molecular activator of NLRP3. Indeed, Lee et al. demonstrated that the murine calcium-sensing receptor activates NLRP3 inflammasome by increasing intracellular calcium and decreasing cyclic adenosine monophosphate (cAMP), suggesting the intimate relationship between calcium signaling and inflammasome activation. Several NLRP3 activators mobilize calcium, thereby promoting mitochondrial damage [86]. Thus, mitochondrial calcium overload induces activation of mitochondrial PTP as well as mtROS generation, which stimulates the release of mtDNA, leading to NLRP3 activation [87, 88].

Mitochondrial matrix calcium overload not only provokes the generation of oxidative stress but also causes the release of Cyt c, resulting in apoptosis [89-92]. For instance, an MCU inhibitor Ru360 significantly decreases amyloidbeta-induced microglial apoptosis, whereas apoptosis is induced by the MCU activator spermine [93]. Similarly, knockdown of endogenous MCU decreases mitochondrial calcium uptake and attenuates apoptosis induced by oxidative stress [80]. When large quantities of calcium are accumulated in the mitochondrial matrix, calcium interacts with cyclophilin D to trigger the opening of the mitochondrial PTP, leading to matrix swelling and outer mitochondrial membrane rupture, and Cyt $c$ release. Moreover, a variety of proapoptotic factors converge on the PTP to control its calcium sensitivity, such as members of the B-cell lymphoma 2 (Bcl-2) family proteins $[94,95]$.

2.4. $m t D N A$. As previously mentioned, $\mathrm{mtDNA}$ is sensitive to mtROS stimuli. Damaged mtDNA can amplify mtROS and trigger oxidative stress through encoding deficient subunits for the respiratory chain, which accelerates the oxidative damage to mitochondrial function until cell death [96]. Therefore, it is possible that mtDNA damage contributes to the pathogenesis induced by oxidative stress [96]. Indeed, a positive relationship between ROS level and mutations in the D-loop region of mtDNA is observed in hepatocarcinoma tissues [97].

mtDNA serves as damage-associated molecular patterns (DAMPs) when released into cytosol or circulation. Lesions and release of mtDNA can trigger the inflammatory response through the Toll-like receptor 9 and cyclic GMP-AMP synthase- (cGAS-) stimulator of interferon genes (STING) [98-100]. For instance, matrix mtDNA release to cytosolic activates cGAS-STING signaling and type I interferon synthesis, which induces cell death-associated inflammation [101]. Damaged mtDNA escaping into the cytosol could activate ERK1/2, PI3K/Akt, tuberin, and mTOR via cGASSTING, leading to increased expression of inflammatory mediators [102]. In contrast, STING knockdown or pretreatment with BAY11-7082 (an NF- $\kappa \mathrm{B}$ inhibitor) attenuates mtDNA-induced TNF- $\alpha$ and IL-6 expression in cultured Kupffer cells [103]. mtDNA activates NLRP3 inflammasome by mechanisms that involve calcium influx and mtROS generation [104]. Furthermore, the release of oxidized mtDNA into the cytosol, where it directly bounds to and activates the NLRP3 inflammasome, stimulates IL-1 $\beta$ secretion [105]. In addition, mtDNA damage activates NOD-like receptor family CARD domain-containing protein 4 inflammasome [106].

Damaged mtDNA has been implicated in cell survival and apoptosis [101, 107]. Ding et al. indicated that mtROS generation during a proinflammatory state induces mtDNA damage and enhances the expression of proprotein convertase subtilisin/kexin type 9 (PCSK9). Between damaged mtDNA and PCSK9 expression manifest a bidirectional cross-talk through p38 MAPK and mTOR activation. Simultaneously, elevated PCSK9 levels induce cell apoptosis by stimulating caspase-3 [108]. mtDNA damage and disordered calcium homeostasis are the main mechanisms for $\mathrm{CdCl}_{2}$ induced apoptosis [107]. Conversely, an increase in the mtDNA copy number via transfecting with an adenoassociated virus vector containing mitochondrial transcription factor A could protect hair cells and HEI-OC1 cells against drug-induced apoptosis. Activation of cytosolic cGAS-STING signaling pathway by mtDNA release could cause pyroptosis, a proinflammatory type of cell death [101, 109]. In addition, mitochondrial inner membrane permeabilization opening can occur during cell death following BAX/BAK-dependent mitochondrial outer membrane permeabilization. This enables the cytosolic release of mtDNA, and inner membrane permeabilization underpins the immunogenic effects of caspase-independent cell death [101]. Hence, matrix mtDNA release from the inner membrane plays a vital role in mitochondrial permeabilizationmediated cell death.

2.5. Dysregulated Mitophagy. Mitophagy is a selective and adaptive response and plays a critical role in surveilling mitochondrial quality and preventing the accumulation of dysfunctional mitochondria. Mammalian mitophagy is induced by two main pathways, specifically damageinduced mitophagy or developmental-induced mitophagy [110]. Damage-induced mitophagy is induced by two main proteins: (i) serine/threonine-protein kinase PINK1 that encodes the PTEN-induced putative kinase and (ii) Parkin (namely, PARK2) that is an E3 ubiquitin protein ligase [111]. Under normal conditions, PINK1 is targeted to the mitochondria, subsequently degraded by matrix processing peptidases and presenilin-associated rhomboid like. After the loss of MMP or the accumulation of misfolded proteins, PINK1 is stabilized on the outer mitochondrial membrane, promoting activation of autophagy recruitment machinery and triggering mitochondria degradation [112]. There are two different recruitment pathways regarding PINK1mediated mitophagy. The canonical pathway is dominated by Parkin ubiquitination of mitochondrial protein. PINK1 is recruited on the outer mitochondrial membrane and phosphorylates ubiquitin to induce the recruitment and phosphorylation of E3 ubiquitin ligase Parkin, leading to the formation of autophagosomes. In this pathway, PINK1 has a small initiator role with the main function being to bring Parkin to the mitochondria. PINK1 can also recruit NDP52 and optineurin to mitochondria to activate mitophagy directly, independently of Parkin [113]. These are required to preserve the LC3-interacting region containing p62, which serves as an adaptor molecule to recruit autophagosome 
membranes in mitochondria. On the other hand, developmental process-induced mitophagy can be induced by the proapoptotic protein, Nip3-like protein X (Nix, also known as Bnip3L), and adenovirus E1B $19 \mathrm{kDa}$-interacting protein 3 (Bnip3), a member of the Bcl-2 family [114]. They prefer to eliminate mitochondrial population during differentiation, rather than remove unhealthy mitochondria [110]. FUN14 domain-containing protein 1 (FUNDC1) is a novel mitophagy receptor and governs mitochondrial turnover via the interaction with recruiting light chain 3 (LC3) to mitochondria [115]. FUNDC1-mediated mitophagy has been described in myocardial ischemia-reperfusion injury, acute kidney injury, and alcohol-related liver disease, attenuating hypoxia-related cardiomyocyte death and inhibiting the progression of diseases [115-117].

Dysregulated mitophagy has been involved in oxidative stress, inflammation, and apoptosis [118-120]. Resveratrol decreases oxidative stress by restoring mitophagy, while knocking down PINK1 reverses resveratrol-mediated ROS reduction [121]. Similarly, mitoquinone activates mitophagy through mitochondrial PINK1/Parkin/PHB2/LC3II pathways and attenuates oxidative stress and neuronal death after subarachnoid hemorrhage in rats [122]. Knockdown of Parkin increases $\mathrm{H}_{2} \mathrm{O}_{2}$-induced oxidative stress [123]. Autophagy/mitophagy-deficient cells with Bnip3 deletion display higher cellular ROS generation compared to the control group during myoblast differentiation. Decreased mitophagy results in the accumulation of damaged mitochondria with a greater propensity for apoptotic cell death signaling in the myoblast throughout differentiation [118].

Removal of damaged mitochondria and excessive ROS blocks the activation of mitochondrial dysfunction-induced inflammasomes and the release of inflammatory cytokines [124]. Both Parkin ${ }^{-1-}$ and PINK1 ${ }^{-1-}$ mice have an increased inflammatory phenotype following exhaustive exercise [125, 126]. During contrast-induced acute kidney injury, mitochondrial damage, mtROS, and subsequent NLRP3 inflammasome activation are more severe in PINK1- or PARK2-deficient mice compared to the wild-type (WT) group [127]. Circulating mtDNA levels and ratios of $\mathrm{mtDNA}$ to nuclear DNA are higher in 40-week-old Parkin ${ }^{-1-}$ mice compared with WT mice, demonstrating that Parkin and PINK1 prevent inflammation by clearing damaged mitochondria [128]. Macrophage-specific p62 ablation reduces mitophagy but causes pronounced accumulation of damaged mitochondria and excessive IL- $1 \beta$-dependent inflammation [129]. Upon LPS stimulation, knockdown of PINK1 via transfection with PINK1 siRNA in macrophages induces the accumulation of dysfunctional mitochondria compared with the control group.

The level of active caspase- 3 increases in cells deficient of PINK1 during LPS stimulation [130]. Matrine represses mitophagy via blocking the PINK1/Parkin pathways, thereby inducing mitochondrial dysfunction and apoptosis in HepG2 cells. Reactivation of mitophagy by the overexpression of Parkin abolishes the proapoptotic effects of matrine on HepG2 cells [131]. Similarly, inhibition of mitophagy by PINK1 or Parkin deficiency increases silibinin-induced breast cancer cell apoptosis, suggesting that mitophagy induced by silibinin treatment serves as a cytoprotective effect, leading to reduction of apoptosis of cancer cells [132]. The roles of mitophagy in apoptosis are cell- and tissue-specific in cancer vs. noncancer tissues.

\section{Mitochondrial Dysregulation in Chronic Respiratory Diseases}

As aforementioned, abnormal mtROS generation, reduced MMP, mitochondrial calcium overload, mtDNA mutation, and dysregulated mitophagy play important roles in modulating oxidative stress, inflammation, apoptosis, and senescence. These cellular processes are associated with the development of chronic respiratory diseases, such as COPD, pulmonary fibrosis, and asthma $[2,16,17]$. Here, we discuss the role of mitochondrial dysfunction in the pathogenesis of these diseases.

3.1. COPD. COPD is one of the most common chronic diseases in the world. This disease encompasses emphysema, chronic bronchitis, and small airway obstruction, where cigarette smoking (CS) is a high-risk factor [133]. Although CS has been identified as the major risk factor for COPD, $15-20 \%$ of all smokers develop COPD, which may be attributed to genetic susceptibility [134]. Long-term CS exposure induces strong and persistent structural changes in mitochondria from COPD epithelium [135]. Here, we discuss the involvement of mitochondrial dysfunction in the pathogenesis of COPD in terms of mtROS, MMP, mtDNA, mitochondrial calcium, and mitophagy [136].

3.1.1. $m t R O S$. Increased $m t R O S$ and decreased MnSOD levels are observed in mitochondria isolated from bronchial biopsies from COPD patients compared to healthy neverand ex-smokers [137]. Similarly, human airway smooth muscle cells isolated from patients with COPD have reduced mitochondrial complex, ATP content, and basal and maximum respiration and increased mtROS compared with those from healthy control subjects [138]. Production of $\mathrm{H}_{2} \mathrm{O}_{2}$ by isolated mitochondria from the vastus lateralis is significantly higher in patients with COPD than control subjects with normal lung function, and complex III is identified as the main mitochondrial site of excess ROS production in skeletal muscle of patients with COPD [139]. CS extract (CSE) increases mitochondrial elongation/fragmentation and mtROS and reduces ATP levels in lung epithelial cells and fibroblasts [140]. Pulmonary vascular endothelial barrier injury and inflammation are important pathophysiological processes in CSE-induced COPD. Human umbilical vein endothelial cells treated with MitoQ protect against CSEinduced endothelial barrier injury and inflammation [141]. The iron-responsive element-binding protein 2 (IRP2), as a regulator of mitochondrial function, increases mitochondrial iron loading and levels of Cyt c oxidase, which leads to mitochondrial dysfunction and subsequent experimental COPD. Mice deficient in IRP2 are protected from CS-induced experimental COPD. In vitro, human airway epithelial cells deficient in IRP2 are protected from CSE-induced cell death and mtROS. In contrast, mitochondrial iron chelation 
alleviates CS-induced mtROS, bronchitis, and emphysema in mice with established COPD [142].

3.1.2. MMP. Decreased MMP is observed in mitochondria isolated from bronchial biopsies from COPD patients compared to healthy never- and ex-smokers [137]. Human airway smooth muscle cells from patients with COPD also have reduced MMP compared with those from healthy control subjects [138]. When the epithelial cells are treated with $10 \%$ CSE, MMP and ATP production is significantly reduced, whereas ROS and apoptosis are elevated [143]. Compared with normal human bronchial epithelial cells, CS solution- and PM2.5-CS solution-treated cells show a significant loss of MMP, whereas PM2.5-CS solution-treated human bronchial epithelial cells than CS solution cells show a greater loss of MMP. Meanwhile, caspase activities increase significantly in CS solution-treated cells and increase more dramatically in PM2.5-CS-treated cells. This provides a new idea about the mechanism of PM2.5 on COPD, and inferred cigarette-inflamed airways are more sensitive to PM2.5 than normal airways [144]. In addition, compared with the control group, MMP and ATP levels in the quadriceps muscle are significantly reduced in CS-exposed rats, while apoptosis is increased. Compared with the model group, MMP and ATP levels increase significantly in the Bufei Jianpi and aminophylline groups, while apoptosis is lower. These findings suggest that Bufei Jianpi granules attenuate mitochondrial dysfunction via increasing MMP and ATP levels and subsequently alleviate cell apoptosis in peripheral muscles and the development of COPD [145].

3.1.3. Mitochondrial Calcium. ER depletion of calcium storage is decreased in patients with COPD, and the calcium release from the ER is significantly decreased in epithelial cells from smokers (regardless of COPD status). Calcium release is clearly impaired in smokers with COPD, whereas smokers without COPD seem to be protected from the action of CS on ER calcium release. However, they have not identified the specific calcium pathway affected by COPD, and the changes of mitochondrial calcium remain unclear [146]. Intracellular calcium levels are increased in human lymphocytes in patients with COPD as compared to nonsmokers and smokers without COPD. H-DHPM, a novel calcium channel blocker, treated cells show a decrease in the intracellular calcium level as compared to the control cells and restore endothelial nitric oxide synthase expression in lymphocytes from COPD patients [147]. To date, the changes of mitochondrial calcium influx in the pathological progress of COPD are unclear, which needs to be further investigated.

3.1.4. $m t D N A$. Zhang et al. evaluated the association of plasma mtDNA (p-mtDNA) with COPD severity and progression in the SPIROMICS cohort with 700 subjects [148]. They found that $\mathrm{p}$-mtDNA levels have no significant differences between nonsmokers and ever smokers without COPD. Compared to nonsmokers and smokers without airflow obstruction, p-mtDNA levels are higher in mild or moderate COPD subjects. Notably, severe COPD participants have lower $\mathrm{p}$-mtDNA levels compared with mild or moderate COPD participants. This indicates that mitochondrial dysfunction may play a role in the transition from mild to severe disease in COPD. In addition, the p-mtDNA levels have sex differences in subgroup analysis, a difference remaining significant after adjustment for age and smoking status [148]. Another study found that urine mtDNA and p-mtDNA available on the same patients did not linearly associate in the SPIROMICS cohort, suggesting that urine mtDNA is not simply a result of circulating p-mtDNA filtered by the kidney. This may have entirely different sources and represents distinct biological phenomena [149]. Specifically, urine mtDNA levels are measured and compared in the following four groups, including the never smokers, smokers without airflow obstruction, mild/moderate COPD, and severe COPD groups. Urine mtDNA levels are associated with increased respiratory symptom burden, especially among smokers without COPD. There are significant sex differences in urine mtDNA levels in patients with COPD. Urine mtDNA is only associated with worse spirometry in male patients with emphysema, while with worse respiratory symptoms in females only. These findings suggest that extracellular mtDNA levels may contribute to identifying distinct clinical phenotypes and underlying pathobiological differences in males versus females with COPD [149].

In addition, patients with asthma-COPD overlap syndrome have an increase in the $\mathrm{mtDNA} / \mathrm{nDNA}$ ratio in the blood [150]. In mice, exposure to CS elevates the levels of DAMPs, reflected by an increase in mtDNA, high mobility group box-1, heat shock protein 70 , and double-stranded DNA, and increases numbers of neutrophils in bronchoalveolar lavage (BAL) fluid, which is statistically reduced upon treatment with necrostatin-1. In vitro, CSE-exposed human bronchial epithelial cells show a significant increase in mtDNA, high mobility group box-1, heat shock protein 70 , and double-stranded DNA release, and elevate in the percentage of necrotic cells. CS exposure induces necrosis of bronchial epithelial cells and subsequent DAMP release, triggering neutrophilic airway inflammation [151].

3.1.5. Mitophagy. Both enhanced and impaired mitophagy have been implicated in COPD pathogenesis. Fibroblasts from patients with COPD show impaired mitophagy, which is associated with increased senescence [140]. Parkin protein levels are decreased in COPD lungs compared with nonCOPD lungs. Reduced Parkin levels lead to the lack of mitophagy, accelerating cellular senescence in bronchial epithelial cells [152]. Parkin protein is the rate-limiting factor in PINK1-Parkin-mediated mitophagy. In comparison to WT mice, Parkin knockout mice have aggravated emphysematous changes, accumulation of damaged mitochondria, and oxidative modifications accompanied by accelerated cellular senescence, following CS exposure. Conversely, induced mitophagy by Parkin overexpression mitigates the progression of COPD [153]. In vitro, mitophagy plays a pivotal role in the removal of CS-induced mitochondrial dysregulation; silencing PINK1 and Parkin aggravates the development of COPD [152]. Impaired mitophagy aggravates CS stressinduced cellular senescence during the development of COPD [140]. These researches indicate that activating 
mitophagy prevents the pathological progression of COPD. However, Mizumura et al. reported that lung epithelial cells isolated from COPD patients display increased expression of PINK1. PINK1 deletion protects against CS-induced mitochondrial dysfunction, airspace enlargement, and mucociliary clearance in mice. Genetic deficiency of PINK1 and the mitochondrial division/mitophagy inhibitor Mdivi-1 protect against CS-induced necroptosis and mitochondrial dysfunction in vitro [154]. CS could increase Nix protein expression and induce mitophagy. Nix overexpression enhances mitophagy and aggravates mitochondrial dysfunction and cell injury, promoting the progression of COPD [155]. Similarly, FUNDC1 serves as another mitophagy receptor, which is highly expressed in CSE-treated human bronchial epithelial cell line and in vivo CS-induced COPD mouse models. Meanwhile, silencing FUNDC1 reduces levels of IL- 6 and TNF- $\alpha$ and inhibits CSE-induced mitophagy, cell apoptosis, and the progression of COPD [156]. These findings show that mitophagy exhibits a complex role in the pathological processes in COPD. Activated mitophagy plays dual roles in COPD, which is often specific to a particular scenario. Therefore, we conjecture that mitophagy might ascertain the final fate of a cell depending on a complex interaction between several factors, including regulating intracellular ATP levels, initiating autophagic cell death, or triggering other types of cell death by modulating overlapping molecules.

3.2. Pulmonary Fibrosis. Pulmonary fibrosis is a devastating lung disease with a median survival of only 3 years after diagnosis. It is characterized by the accumulation of extracellular matrix proteins in the lung interstitium, leading to distortion of normal lung architecture [157]. Current therapeutic approaches have not been successful in improving disease outcomes [158]. Pulmonary fibrosis is associated with aging, and the median age to diagnose this disease is approximately 66 years [159-161]. Senescent lung fibroblasts from pulmonary fibrosis patients exhibit mitochondrial dysfunction, including disrupted cristae and a diminished capacity for oxidative phosphorylation [162]. Increased superoxide production by dysfunctional mitochondria accelerates senescence in lung fibroblasts by prolonging DNA damage response [163]. Thus, mitochondrial dysfunction plays a critical role in mediating cell senescence and promotes the development of pulmonary fibrosis. Here, we summarize the role played by mitochondrial dysfunction in the development of pulmonary fibrosis, including mtROS, MMP, mtDNA, mitochondrial calcium, and mitophagy.

3.2.1. $m t R O S$. $m$ tROS generation is associated with the development of pulmonary fibrosis. Alveolar macrophages from patients with pulmonary fibrosis have significantly greater mitochondrial $\mathrm{H}_{2} \mathrm{O}_{2}$ produced in the membrane or mitochondrial fractions, while normal subjects show no difference in mitochondrial $\mathrm{H}_{2} \mathrm{O}_{2}$ production. Mitochondria isolated from bleomycin-exposed mice generate increased mitochondrial $\mathrm{H}_{2} \mathrm{O}_{2}$ than saline controls [164]. Compared to WT mice, crocidolite/bleomycin-exposed mitochondrialtargeted catalase (MCAT) mice exhibit reduced pulmonary fibrosis as measured by lung collagen levels and lung fibrosis score. In in vitro, alveolar type II (AT2) cells isolated from WT mice, mtROS production is significantly increased following asbestos exposure. Notably, compared to the WT group, AT2 cells from MCAT mice have significantly decreased levels of asbestos-induced mtROS production. Targeting mtROS levels may be an efficient therapeutic target for preventing pulmonary fibrosis [165]. In vitro, challenge of bronchial airway epithelial cells with bleomycin enhances mitochondrial $\mathrm{O}_{2}^{--}$production. Treatment of bronchial airway epithelial cells with Mito-TEMPO, a specific scavenger of mtROS, decreases mitochondrial $\mathrm{O}_{2}{ }^{--}, \mathrm{mtDNA}$ damage, and apoptosis [166]. Increased mtROS production leads to mtDNA damage and apoptosis of alveolar epithelial cells, which are necessary for the development of pulmonary fibrosis [167]. Similarly, TGF- $\beta 1$ stimulation of alveolar epithelial cells results in mtROS production, and this is reduced by Mito-TEMPO treatment [168].

3.2.2. MMP. Loss of MMP is an important mechanism of the development of pulmonary fibrosis. Lung tissue cells isolated from paraquat-exposed rats have a significant loss of MMP compared with saline controls, while treatment with cyclosporine A, an inhibitor of mitophagy, significantly improves the paraquat-decreased MMP in the lung cells of rats [169]. The change in the MMP has been thought to be viewed as an early event of mitophagy. In vitro, macrophage treatment with bleomycin shows a loss in MMP in a dose-dependent manner [164]. Similarly, A549 cells display increased mitochondrial depolarization following bleomycin treatment [168]. This suggests that reduced MMP participates in the pathogenesis of pulmonary fibrosis.

3.2.3. Mitochondrial Calcium. Alveolar macrophages are the predominant cells in BAL fluid from normal participants and patients with asbestosis. Compared with control subjects, patients with asbestosis have higher levels of mitochondrial calcium [170]. Similar findings have further demonstrated that MCU gene and protein expression are significantly higher in mitochondria of lung macrophages from subjects with pulmonary fibrosis compared to control subjects. In vivo, lung macrophages from bleomycin-injured mice have increased MCU expression. Mitochondrial calcium is markedly reduced in lung macrophages from bleomycin-exposed mice transfected with dominant negative MCU compared to WT controls [171]. Furthermore, these mice are protective from bleomycin-induced pulmonary fibrosis. In vitro, mouse alveolar macrophage cells are transfected with scrambled or MCU siRNA and exposed to asbestos. After asbestos exposure, mitochondrial calcium in MCUsilenced cells is significantly reduced to levels similar to that in unexposed cells. These findings suggest that the increased activity of MCU along with the overload of mitochondrial calcium in lung macrophages promotes the pathogenesis of pulmonary fibrosis [170]. Hence, MCU-mediated imbalanced calcium in mitochondria contributes to fibrotic repair after lung injury.

3.2.4. $m t D N A$. Compared to controls, BAL fluid from pulmonary fibrosis subjects exhibits a robust increase in 
extracellular mtDNA. Likewise, plasma obtained from patients with pulmonary fibrosis shows substantial increases in mtDNA compared with control subjects. Plasma mtDNA concentrations are obtained prior to the initiation of antifibrotic agent pirfenidone therapy and three months after starting therapy. Compared to nonresponders, plasma mtDNA numbers are increased, whereas responders experience a reduction in mtDNA numbers. Excessive plasma mtDNA is predictive of all-cause mortality by a 43-month longitudinal follow-up. These findings indicate that plasma mtDNA measurements might be a useful predictor of treatment efficacy and clinical outcomes in pulmonary fibrosis $[172,173]$.

Sirtuin 3 (SIRT3) primarily localizes in mitochondria and could regulate acetylation of mitochondrial proteins MnSOD and 8-oxoguanine glycosylase, a critical role in the repair of mtDNA oxidative damage. In vivo, compared with WT controls, SIRT3-knockout mice show exacerbated fibrosis after bleomycin exposure. Increased pulmonary fibrosis is associated with decreased levels of 8-oxoguanine glycosylase and concomitant accumulation of oxidized guanine and increased mtDNA damage. Inversely, the transgenic mice with whole-body SIRT3 overexpression are protective against bleomycin-induced mtDNA damage and pulmonary fibrosis. In vitro, TGF- $\beta 1$ treatment decreases the expression of endogenous SIRT3, subsequently increasing mtDNA damage. Conversely, overexpression of SIRT3 by adenovirusmediated transduction reverses the effects of TGF- $\beta 1$ on mtDNA damage and suppresses TGF- $\beta 1$-induced myofibroblast differentiation $[174,175]$.

3.2.5. Mitophagy. Alveolar AT2 cells from pulmonary fibrosis patients exhibit accumulation of dysfunctional mitochondria and impaired autophagy [159]. In vivo, PINK1-deficient mice develop similarly dysmorphic, dysfunctional mitochondria in the AT2 cells and are susceptible to apoptosis and development of pulmonary fibrosis [159]. Similarly, PARK2-knockout mice show enhanced pulmonary fibrosis after bleomycin exposure. This is associated with increased mtROS production and activation of the platelet-derived growth factor receptor (PDGFR)/PI3K/AKT signaling pathway, resulting in increased myofibroblast differentiation and proliferation for fibrotic foci formation [176]. Pirfenidone, an FDA-approved antipulmonary fibrosis drug, triggers mitophagy activation by enhancing PARK2 expression. PARK2 knockdown mice display increased pulmonary fibrosis, and this is efficiently alleviated by pirfenidone [177]. Thyroid hormone improves mitochondrial bioenergetics, promotes mitochondrial biogenesis, and attenuates mitochondria-regulated apoptosis in alveolar epithelial cells. Intriguingly, thyroid hormone does not alleviate the pathological process of pulmonary fibrosis in PINK1-knockout mice [178]. In vitro, knockdown of PINK1 expression in lung epithelial cells results in mitochondria depolarization and expression of profibrotic factors [159]. These results suggest that insufficient mitophagy aggravates the accumulation of dysregulated mitochondria, leading to the development of pulmonary fibrosis. This is in contrast to the findings that mitophagy is required for apoptosis resistance, which activation accelerates the development of pulmonary fibrosis.
Larson-Casey et al. investigated that mitochondria in alveolar macrophages isolated from pulmonary fibrosis patients have higher expression of PINK1, Parkin, and LC3-II compared to control subjects. Likewise, WT mice exposed to bleomycin have enhanced PINK1 and Parkin expression in isolated mitochondria and increased LC3-II in alveolar macrophages. Alveolar macrophages from mice exposed to rapamycin, an autophagy/mitophagy activator, have increased levels of PINK1, Parkin, and p62, as well as LC3-II. Meanwhile, pulmonary fibrosis development is aggravated by rapamycin [164]. In contrast, cyclosporine A serves as an inhibitor of mitophagy, and its treatment mitigates the paraquattreated collagen synthesis in A549 cells. This is attenuated by PINK1 overexpression [169]. The opposite results may ascribe that impaired mitophagy induces oxidative stress injury, but excessive mitophagy prevents apoptosis in a cell-specific manner. Further studies are warranted to investigate the role of FUNDC1, Nix, and Bnip3 in pulmonary fibrosis in terms of mitophagy.

3.3. Asthma. Asthma is a common condition due to chronic inflammation of the lower respiratory tract. Chronic psychosocial stress and environmental exposure such as allergens and air pollutants have been linked to the risk of asthma [179]. Pharmacologic therapies are often not effective in older asthmatic patients and may have more side effects [180]. Cellular, molecular, and animal model studies have revealed that mitochondrial dysregulation is recognized as a key mediator for asthma [181]. A separate study shows that increased bronchial smooth muscle mass in nonsevere asthmatics is associated with exacerbations and worse asthma control in 34 never smoker subjects with nonsevere asthma and 56 nonsevere and 19 severe asthmatics. Meanwhile, the number and the density of mitochondria in bronchial smooth muscle cells are positively correlated to the bronchial smooth muscle area in a subgroup of nonsevere asthmatics. The research further reveals the importance of mitochondria in the progression of asthma [182]. Long-term administration of corticosteroids induces mitochondrial dysfunction and oxidative damage of mitochondria and nuclear DNAs in skeletal muscles $[183,184]$. It remains unknown whether the side effects of long-term corticosteroid therapy in asthma are related to mitochondrial dysfunction.

3.3.1. $m t R O S$. MMP, $\mathrm{mtDNA}$, and $\mathrm{mtROS}$ participate in intercellular communication within the airways of human subjects with asthma [185]. mtROS production is increased in the venous blood of severe asthmatic patients compared with the control group [186]. In the ovalbumin-induced murine asthma model, mtROS production is increased in comparison with saline control. Mito-TEMPO significantly attenuates mtROS, TGF- $\beta$, and collagen deposition in ovalbumin-challenged mice. In vitro, human airway epithelial cells treated with IL13 have an increase in mtROS and collagen expression, and this is attenuated by Mito-TEMPO treatment [187].

3.3.2. MMP. Human peripheral blood eosinophils are purified from self-reported allergic asthma or healthy donors. MMP is reduced in eosinophils from allergic asthma donors 


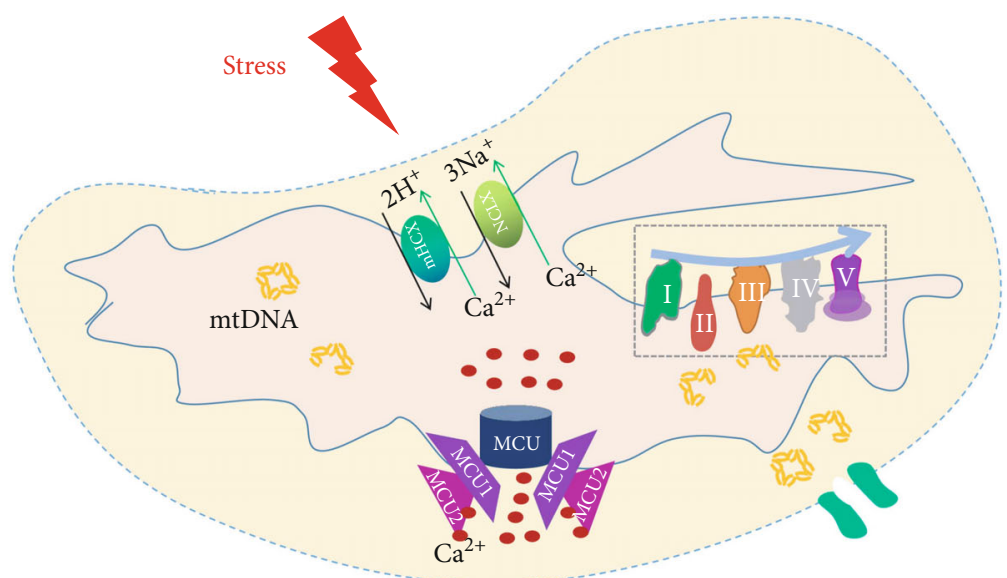

Mitochondrial dysfunction

\begin{tabular}{|c|c|c|c|c|}
\hline mtROS accmulation & Loss of MMP & $\begin{array}{l}\text { Mitochondrial } \\
\text { calcium overload }\end{array}$ & $\begin{array}{l}\text { mtDNA mutations } \\
\text { or releaase }\end{array}$ & $\begin{array}{l}\text { Mitophagy } \\
\text { dysregulation }\end{array}$ \\
\hline
\end{tabular}

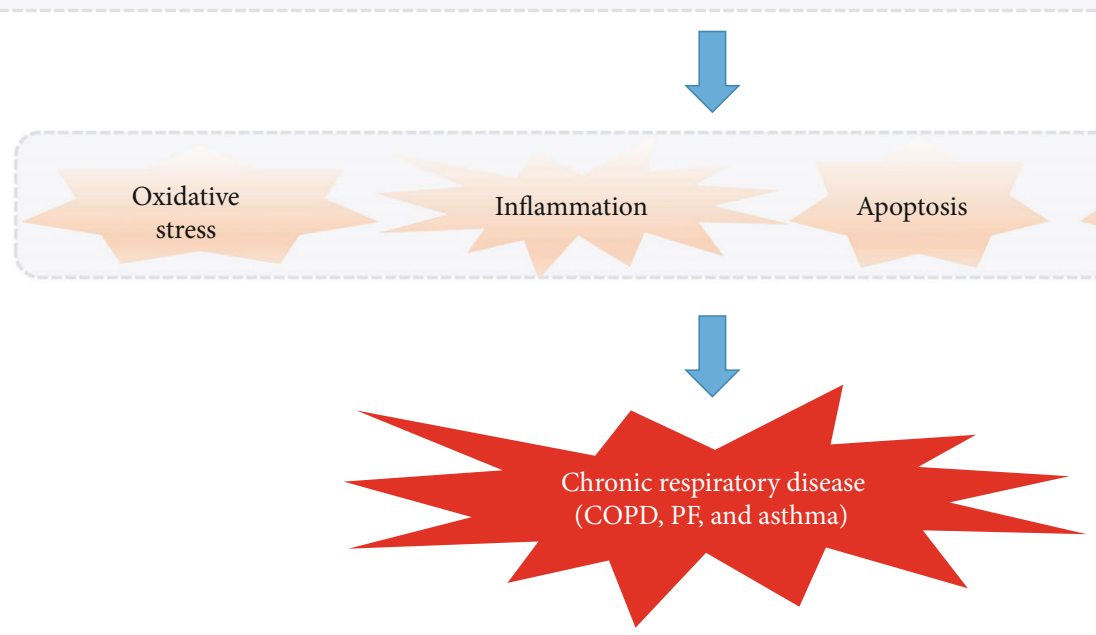

Figure 3: Mitochondrial dysfunction contributes to the development of chronic respiratory diseases. Mitochondrial dysfunction accompanies increased mtROS, reduced MMP, mitochondrial calcium overload, mtDNA damage and release, and abnormal mitophagy. This could cause a series of cellular processes, including oxidative stress, inflammasome activation, apoptosis, and senescence. All these cellular processes participate in the pathogenesis and progression of chronic respiratory diseases, including COPD, pulmonary fibrosis (PF), and asthma.

compared with healthy donors [188]. Compared to the control group, the respiratory control ratio of mitochondria and MMP is significantly lower in the rat asthma models [189]. A separate study discovers that fibroblasts from severe asthmatics have reduced MMP and metabolic activity and increased expression of mitophagy genes, PINK1 and Parkin [190]. Whether rectifying or increasing MMP protects against asthma remains unclear.

3.3.3. Mitochondrial Calcium. Compared to control subjects, mitochondrial mass and oxygen consumption are higher in the bronchial smooth muscle from asthmatic subjects, which is associated with enhanced extracellular calcium influx and mitochondrial biogenesis. This may promote the proliferation of bronchial smooth muscle cells, leading to airway remodeling [191]. A calcium channel blocker gal- lopamil decreases the proliferation of bronchial smooth muscle cells from patients with severe asthma. Gallopamil treatment for 12 months reduces bronchial smooth muscle remodeling and prevents the occurrence of asthma exacerbations [192]. In the ovalbumin model of allergic asthma, MCU knockdown mice show decreased apoptosis within the large airway epithelial cells compared to WT mice. Expression of the tight junction protein zona occludens-1 is not significantly changed in MCU knockdown mice. In contrast, ovalbumin-treated WT mice have significantly reduced zona occludens-1 staining within the airway epithelia. In primary human respiratory epithelial cells, MCU deficiency suppresses mitochondrial calcium uptake and ROS production, maintains MMP, and protects against apoptosis in response to the pleiotropic Th2 cytokine IL-13 [193]. Additionally, allergen-induced ROS generation activates 
calcium/calmodulin-dependent protein kinase II (CaMKII) that plays a role in downstream regulation of autophagy/mitophagy, resulting in hyperresponsiveness, ROS generation, and Th2-associated lung inflammation. Mitochondrial CaMKII inhibition mitigates allergen-induced autophagy/mitophagy, mitochondrial dysfunction, and cytokine production in bronchial epithelial cells and subsequent airway hyperreactivity, inflammation, and asthma [194].

3.3.4. mtDNA. Through analysis of 16,158 mitochondrial single nucleotide polymorphisms, different sex-specific locations in the mitochondrial genome are observed in a human cohort with 372 asthmatic children (aged 5 to 18 years, 35\% female and $65 \%$ male) and 395 healthy children (aged 5 to 18 years, $44 \%$ female and $56 \%$ male) [195]. One of the genetic causes of asthma in girls could be a dysfunction of the MTND2 and MT-RNR2 genes. In boys, variants in the CYB gene provoke changes in ROS production and could be causative of asthma [195]. Carpagnano et al. enrolled fifty-three patients with severe asthma, 11 patients with mildmoderate asthma, and 12 healthy subjects and measure the content of mtDNA and nuclear DNA (nDNA) in exhaled breath condensate. Higher exhaled mtDNA/nDNA is observed in severe asthmatics, respectively, compared to mild-moderate ones and to healthy controls [196].

To establish an in vivo model of asthma, WT and Parkin deficiency mice are intranasally inoculated with IL-13 or house dust mites. WT mice show significantly increased numbers of both neutrophils and eosinophils in BAL fluid following IL-13 treatment than Parkin deficiency mice. Treatment with IL-13 also increases mtDNA release and neutrophil chemokine levels in BAL fluid of WT mice compared with Parkin deficiency mice. In vitro, Parkin deficiency in IL-13-stimulated human tracheobronchial epithelial cells inhibits mtDNA release. This suggests a link of mitophagy, mtDNA, and inflammation in the pathogenesis of asthma [197]. Increased accumulation of cytosolic double-stranded DNA is observed in the airway epithelium of ovalbumin- or house dust mite-exposed mice and in human bronchial epithelial cells treated with IL-33. Interestingly, Mito-TEMPO could reduce IL-33-induced cytoplasmic double-stranded DNA accumulation in human bronchial epithelial cells, which is possibly associated with suppressing the release of mtDNA into the cytosol [198]. Altogether, mtDNA damage or release plays important roles in the pathogenesis of asthma.

3.3.5. Mitophagy. PINK1/Parkin-mediated mitophagy is enhanced from severe asthmatic patients compared to healthy controls by bioinformatics analysis [190]. Brushed bronchial epithelial cells isolated from patients with asthma have significantly higher Parkin mRNA levels than healthy controls [197]. Additionally, Bnip3 expression is increased in airway smooth muscle cells from asthmatic donors compared to that from control donors. Silencing Bnip3 in human airway smooth muscle cells alleviates cell adhesion, migration, and proliferation and suppresses the development of asthma [199]. Moreover, there is a significant upregulation in the expression of LC3B and ATG5 in the lung tissues of asthmatic patients compared to healthy controls. In vivo, a cockroach extract-established asthmatic mouse model also shows significantly increased expression of LC3B and ATG5 in the lung tissues. In vitro, human bronchial epithelial cells show induction of mitophagy following cockroach allergen than controls. These results indicate that excessive activation of mitophagy plays an important mechanism in developing asthma [194]. Further study is required to determine the role of FUNDC1 and Nix in modulating mitophagy during the development of asthma.

\section{Conclusion and Future Directions}

Although mitochondria are indispensable for energy metabolism and play important roles in modulating cell signaling and maintaining cell function and tissue homeostasis. Normal mitochondria are sustained by stabilization of MMP, balance of mitochondrial calcium, integrity of mtDNA, and timely clearance of damaged mitochondria via mitophagy. Mitochondrial dysfunction participates in the pathogenesis of COPD, pulmonary fibrosis, and asthma via mtROS overproduction, mtDNA damage and release, reduced MMP, and abnormal mitophagy (Figure 3). Targeting dysfunctional mitochondria could be a promising approach to prevent or halt the development of these chronic lung diseases.

Mitochondria are dynamic through fission and fusion. Quantitative imaging-based assessment of mitochondrial morphology and dynamics using the full 3D mitochondrial network with an extension to $4 \mathrm{D}$ analysis can provide valuable insights into lung cellular physiology and pathophysiology of these chronic lung diseases [200]. Moreover, there are many different cells in the lung. With the progress of technologies, single-cell RNA sequencing may provide directions on mitochondrial dysfunction in a cell-specific manner. A recent report showed that somatic mutations in mtDNA can be used as natural genetic barcodes to explore cellular states and clonal dynamics using single-cell sequencing technologies [201]. This has three key advantages, including being highly scalable, directly applicable to human tissues, and combined with assays to profile a cell's state at the chromatin or transcriptome level. To date, using single-cell RNA sequencing analysis to investigate the mechanism of mitochondrial dysfunction for chronic respiratory diseases has not been reported. In addition, using a newly developed digital spatial profiling platform (NanoString nCounter and GeoMX platforms), the temporal and spatial heterogeneity of mitochondrial biology and dysfunction could be dissected in these diseases. All those may open up novel avenues to further explore the pathogenesis progress of these diseases.

\section{Conflicts of Interest}

The authors declare no conflicts of interest, financial or otherwise.

\section{Authors' Contributions}

Conception and design are performed by HY, drafting the manuscript was performed by WZ and JQ, and revising the manuscript was performed by YS and SX. All authors have 
read and agreed to the published version of the manuscript. Wen-cheng Zhou and Jiao Qu contributed equally to this paper. Wen-cheng Zhou and Jiao Qu are co-first authors.

\section{Acknowledgments}

This research was funded by Zhejiang Grants Funded by the Pharmaceutical Association Hospital Pharmacy Special Scientific Researches (No. 2020ZYY18).

\section{References}

[1] A. Rongvaux, "Innate immunity and tolerance toward mitochondria," Mitochondrion, vol. 41, pp. 14-20, 2018.

[2] L. Yue and H. Yao, "Mitochondrial dysfunction in inflammatory responses and cellular senescence: pathogenesis and pharmacological targets for chronic lung diseases," British Journal of Pharmacology, vol. 173, no. 15, pp. 2305-2318, 2016.

[3] P. Mishra and D. C. Chan, "Mitochondrial dynamics and inheritance during cell division, development and disease," Nature Reviews Molecular Cell Biology, vol. 15, no. 10, pp. 634-646, 2014.

[4] A. W. el-Hattab and F. Scaglia, "Mitochondrial DNA depletion syndromes: review and updates of genetic basis, manifestations, and therapeutic options," Neurotherapeutics, vol. 10, no. 2, pp. 186-198, 2013.

[5] M. Akbari, T. B. L. Kirkwood, and V. A. Bohr, "Mitochondria in the signaling pathways that control longevity and health span," Ageing Research Reviews, vol. 54, article 100940, 2019.

[6] I. J. Holt and A. Reyes, "Human mitochondrial DNA replication," Cold Spring Harbor Perspectives in Biology, vol. 4, no. 12, 2012.

[7] T. J. Nicholls, C. A. Nadalutti, E. Motori et al., "Topoisomerase $3 \alpha$ Is Required for Decatenation and Segregation of Human mtDNA," Molecular Cell, vol. 69, no. 1, pp. 923.e6, 2018.

[8] M. Uittenbogaard and A. Chiaramello, "Mitochondrial biogenesis: a therapeutic target for neurodevelopmental disorders and neurodegenerative diseases," Current Pharmaceutical Design, vol. 20, pp. 5574-5593, 2014.

[9] J. L. Chiang, P. Shukla, K. Pagidas et al., "Mitochondria in ovarian aging and reproductive longevity," Ageing Research Reviews, vol. 63, article 101168, 2020http://www.ncbi.nlm .nih.gov/pubmed/32896666.

[10] S. L. Archer, "Mitochondrial dynamics-mitochondrial fission and fusion in human diseases," New England Journal of Medicine, vol. 369, pp. 2236-2251, 2013, http://www.ncbi.nlm.nih .gov/pubmed/24304053.

[11] J. Piquereau, F. Caffin, M. Novotova et al., "Mitochondrial dynamics in the adult cardiomyocytes: which roles for a highly specialized cell?," Frontiers in Physiology, vol. 4, p. 102, 2013, https://www.ncbi.nlm.nih.gov/pubmed/ 23675354.

[12] E. M. Mejia, H. Nguyen, and G. M. Hatch, "Mammalian cardiolipin biosynthesis," Chemistry and Physics of Lipids, vol. 179, pp. 11-16, 2014, https://www.ncbi.nlm.nih.gov/ pubmed/24144810.

[13] S. Marchi, S. Patergnani, S. Missiroli et al., "Mitochondrial and endoplasmic reticulum calcium homeostasis and cell death," Cell Calcium, vol. 69, pp. 62-72, 2018, http://www .ncbi.nlm.nih.gov/pubmed/28515000.

[14] Y. Yang, S. Karakhanova, W. Hartwig et al., "Mitochondria and mitochondrial ros in cancer: novel targets for anticancer therapy," Journal of Cellular Physiology, vol. 231, pp. 25702581, 2016, http://www.ncbi.nlm.nih.gov/pubmed/26895995.

[15] Y. S. Prakash, C. M. Pabelick, and G. C. Sieck, "Mitochondrial dysfunction in airway disease," Chest, vol. 152, pp. 618-626, 2017, http://www.ncbi.nlm.nih.gov/pubmed/28336486.

[16] J. L. Larson-Casey, C. He, and A. B. Carter, "Mitochondrial quality control in pulmonary fibrosis," Redox Biology, vol. 33, article 101426, 2020.

[17] S. M. Cloonan, K. Kim, P. Esteves, T. Trian, and P. J. Barnes, "Mitochondrial dysfunction in lung ageing and disease," European Respiratory Review, vol. 29, 2020http://www.ncbi .nlm.nih.gov/pubmed/33060165.

[18] A. O. Oyewole and M. A. Birch-Machin, "Mitochondria-targeted antioxidants," The FASEB Journal, vol. 29, pp. 47664771, 2015, http://www.ncbi.nlm.nih.gov/pubmed/26253366.

[19] P. R. Angelova and A. Y. Abramov, "Role of mitochondrial ros in the brain: from physiology to neurodegeneration," FEBS Letters, vol. 592, pp. 692-702, 2018, http://www.ncbi .nlm.nih.gov/pubmed/29292494.

[20] M. S. Carraway, H. B. Suliman, C. Kliment, K. E. Welty-Wolf, T. D. Oury, and C. A. Piantadosi, "Mitochondrial biogenesis in the pulmonary vasculature during inhalational lung injury and fibrosis," Antioxidants \& Redox Signaling, vol. 10, pp. 269-275, 2008, http://www.ncbi.nlm.nih.gov/pubmed/ 17999632.

[21] R. M. Whitaker, D. Corum, C. C. Beeson, and R. G. Schnellmann, "Mitochondrial biogenesis as a pharmacological target: a new approach to acute and chronic diseases," Annual Review of Pharmacology and Toxicology, vol. 56, pp. 229249, 2016, http://www.ncbi.nlm.nih.gov/pubmed/26566156.

[22] E. Sedlak and A. Musatov, "Inner mechanism of protection of mitochondrial electron-transfer proteins against oxidative damage. Focus on hydrogen peroxide decomposition," Biochimie, vol. 142, pp. 152-157, 2017, http://www.ncbi.nlm .nih.gov/pubmed/28918334.

[23] P. S. Brookes, Y. Yoon, J. L. Robotham, M. W. Anders, and S.-S. Sheu, "Calcium, atp, and ros: a mitochondrial love-hate triangle," American Journal of Physiology-Cell Physiology, vol. 287, pp. C817-C833, 2004, http://www .ncbi.nlm.nih.gov/pubmed/15355853.

[24] D. B. Zorov, C. R. Filburn, L.-O. Klotz, J. L. Zweier, and S. J. Sollott, "Reactive oxygen species (ros)-induced ros release: a new phenomenon accompanying induction of the mitochondrial permeability transition in cardiac myocytes," Journal of Experimental Medicine, vol. 192, pp. 1001-1014, 2000, http:// www.ncbi.nlm.nih.gov/pubmed/11015441.

[25] S. S. Korshunov, O. V. Korkina, E. K. Ruuge, V. P. Skulachev, and A. A. Starkov, "Fatty acids as natural uncouplers preventing generation of $\mathrm{O} 2$.- and $\mathrm{H} 2 \mathrm{O} 2$ by mitochondria in the resting state," FEBS Letters, vol. 435, pp. 215-218, 1998, http://www.ncbi.nlm.nih.gov/pubmed/9762912.

[26] D. B. Zorov, M. Juhaszova, and S. J. Sollott, "Mitochondrial reactive oxygen species (ros) and ros-induced ros release," Physiological Reviews, vol. 94, pp. 909-950, 2014, http:// www.ncbi.nlm.nih.gov/pubmed/24987008.

[27] Y. Quan, Y. Xin, G. Tian, J. Zhou, and X. Liu, "Mitochondrial ros-modulated mtdna: a potential target for cardiac aging," 
Oxidative Medicine and Cellular Longevity, vol. 2020, Article ID 9423593, 11 pages, 2020.

[28] P. T. Schumacker, M. N. Gillespie, K. Nakahira et al., "Mitochondria in lung biology and pathology: more than just a powerhouse," American Journal of Physiology. Lung Cellular and Molecular Physiology, vol. 306, pp. L962-L974, 2014, http://www.ncbi.nlm.nih.gov/pubmed/24748601.

[29] D. C. Zank, M. Bueno, A. L. Mora, and M. Rojas, "Idiopathic pulmonary fibrosis: aging, mitochondrial dysfunction, and cellular bioenergetics," Frontiers in Medicine, vol. 5, p. 10, 2018, http://www.ncbi.nlm.nih.gov/pubmed/ 29459894.

[30] R. Zhou, A. S. Yazdi, P. Menu, and J. Tschopp, "A role for mitochondria in nlrp3 inflammasome activation," Nature, vol. 469, pp. 221-225, 2011, http://www.ncbi.nlm.nih.gov/ pubmed/21124315.

[31] S. Alfonso-Loeches, J. R. Urena-Peralta, M. J. MorilloBargues, J. O. De La Cruz, and C. Guerri, "Role of mitochondria ros generation in ethanol-induced nlrp3 inflammasome activation and cell death in astroglial cells," Frontiers in Cellular Neuroscience, vol. 8, p. 216, 2014, http://www.ncbi.nlm.nih.gov/pubmed/25136295.

[32] Y. Han, X. Xu, C. Tang et al., "Reactive oxygen species promote tubular injury in diabetic nephropathy: the role of the mitochondrial ros-txnip-nlrp3 biological axis," Redox Biology, vol. 16, pp. 32-46, 2018.

[33] X. Yao, D. Carlson, Y. Sun et al., "Mitochondrial ros induces cardiac inflammation via a pathway through mtdna damage in a pneumonia-related sepsis model," PLoS One, vol. 10, article e0139416, 2015http://www.ncbi.nlm.nih.gov/pubmed/ 26448624.

[34] H. A. Elfawy and B. Das, "Crosstalk between mitochondrial dysfunction, oxidative stress, and age related neurodegenerative disease: etiologies and therapeutic strategies," Life Sciences, vol. 218, pp. 165-184, 2019, http://www.ncbi.nlm.nih .gov/pubmed/30578866.

[35] Z. Ungvari, S. Tarantini, A. J. Donato, V. Galvan, and A. Csiszar, "Mechanisms of vascular aging," Circulation Research, vol. 123, pp. 849-867, 2018, http://www.ncbi.nlm .nih.gov/pubmed/30355080.

[36] H. Jiang, P. Qu, J. W. Wang, G. H. Li, and H. Y. Wang, "Effect of nf-kappab inhibitor on toll-like receptor 4 expression in left ventricular myocardium in two-kidney-one-clip hypertensive rats," European Review for Medical and Pharmacological Sciences, vol. 22, pp. 3224-3233, 2018, http://www.ncbi .nlm.nih.gov/pubmed/29863269.

[37] E. Vringer and S. W. G. Tait, "Mitochondria and inflammation: cell death heats up," Frontiers in Cell and Development Biology, vol. 7, p. 100, 2019, http://www.ncbi.nlm.nih.gov/ pubmed/31316979.

[38] X. Li, F. Fang, Y. Gao et al., "Ros induced by killerred targeting mitochondria (mtkr) enhances apoptosis caused by radiation via cyt c/caspase-3 pathway," Oxidative Medicine and Cellular Longevity, vol. 2019, Article ID 4528616, 11 pages, 2019, http://www.ncbi.nlm.nih.gov/pubmed/30984335.

[39] A. Ruiz-Ramirez, M. A. Barrios-Maya, O. Lopez-Acosta, D. Molina-Ortiz, and M. El-Hafidi, "Cytochrome c release from rat liver mitochondria is compromised by increased saturated cardiolipin species induced by sucrose feeding," American Journal of Physiology. Endocrinology and Metabolism, vol. 309, pp. E777-E786, 2015, http://www.ncbi.nlm .nih.gov/pubmed/26353385.
[40] J. Li, W. He, B. Liao, and J. Yang, "Ffa-ros-p53-mediated mitochondrial apoptosis contributes to reduction of osteoblastogenesis and bone mass in type 2 diabetes mellitus," Scientific Reports, vol. 5, article 12724, 2015http://www.ncbi .nlm.nih.gov/pubmed/26226833.

[41] C.-W. Lee, F.-L. Yen, H.-H. Ko et al., "Cudraflavone c induces apoptosis of a375.S2 melanoma cells through mitochondrial ros production and mapk activation," International Journal of Molecular Sciences, vol. 18, 2017http://www.ncbi.nlm.nih .gov/pubmed/28703746.

[42] D.-F. Dai, P. S. Rabinovitch, and Z. Ungvari, "Mitochondria and cardiovascular aging," Circulation Research, vol. 110, pp. 1109-1124, 2012, http://www.ncbi.nlm.nih.gov/ pubmed/22499901.

[43] T. E. S. Kauppila, J. H. K. Kauppila, and N.-G. Larsson, "Mammalian mitochondria and aging: an update," Cell Metabolism, vol. 25, pp. 57-71, 2017, http://www.ncbi.nlm .nih.gov/pubmed/28094012.

[44] D. F. Dai, L. F. Santana, M. Vermulst et al., "Overexpression of catalase targeted to mitochondria attenuates murine cardiac aging," Circulation, vol. 119, no. 21, pp. 2789-2797, 2009.

[45] D. V. Ziegler, C. D. Wiley, and M. C. Velarde, "Mitochondrial effectors of cellular senescence: beyond the free radical theory of aging," Aging Cell, vol. 14, pp. 1-7, 2015, http://www.ncbi .nlm.nih.gov/pubmed/25399755.

[46] S. Macip, M. Igarashi, L. Fang et al., "Inhibition of p21mediated ros accumulation can rescue p21-induced senescence," The EMBO Journal, vol. 21, no. 9, pp. 2180-2188, 2002.

[47] A. Takahashi, N. Ohtani, K. Yamakoshi et al., "Mitogenic signalling and the p16ink4a-rb pathway cooperate to enforce irreversible cellular senescence," Nature Cell Biology, vol. 8, pp. 1291-1297, 2006, http://www.ncbi.nlm.nih.gov/ pubmed/17028578.

[48] J. Marín-García, "Mitochondrial DNA repair: a novel therapeutic target for heart failure," Heart Failure Reviews, vol. 21, pp. 475-487, 2016, http://www.ncbi.nlm.nih.gov/ pubmed/26940911.

[49] M. Vermulst, J. Wanagat, G. C. Kujoth et al., "DNA deletions and clonal mutations drive premature aging in mitochondrial mutator mice," Nature Genetics, vol. 40, pp. 392-394, 2008, http://www.ncbi.nlm.nih.gov/pubmed/18311139.

[50] H. Rottenberg and J. B. Hoek, "The path from mitochondrial ros to aging runs through the mitochondrial permeability transition pore," Aging Cell, vol. 16, pp. 943-955, 2017, http://www.ncbi.nlm.nih.gov/pubmed/28758328.

[51] C. Ho, E. van der Veer, O. Akawi, and J. G. Pickering, "Sirt1 markedly extends replicative lifespan if the nad+ salvage pathway is enhanced," FEBS Letters, vol. 583, pp. 30813085, 2009, http://www.ncbi.nlm.nih.gov/pubmed/19716821.

[52] L. D. Zorova, V. A. Popkov, E. Y. Plotnikov et al., "Mitochondrial membrane potential," Analytical Biochemistry, vol. 552, pp. 50-59, 2018.

[53] C. M. Palmeira and A. P. Rolo, "Mitochondrial membrane potential (deltapsi) fluctuations associated with the metabolic states of mitochondria," Methods in Molecular Biology, vol. 810, pp. 89-101, 2012, http://www.ncbi.nlm.nih.gov/ pubmed/22057562.

[54] G. Bagkos, K. Koufopoulos, and C. Piperi, "A new model for mitochondrial membrane potential production and storage," 
Medical Hypotheses, vol. 83, pp. 175-181, 2014, http://www .ncbi.nlm.nih.gov/pubmed/24907229.

[55] P. Bernardi, "The mitochondrial permeability transition pore: a mystery solved?," Frontiers in Physiology, vol. 4, p. 95, 2013, http://www.ncbi.nlm.nih.gov/pubmed/ 23675351.

[56] K. Yamakawa, H. Ogura, T. Koh et al., "Platelet mitochondrial membrane potential correlates with severity in patients with systemic inflammatory response syndrome," Journal of Trauma and Acute Care Surgery, vol. 74, pp. 411-417, 2013, http://www.ncbi.nlm.nih.gov/pubmed/23354232.

[57] E. Gottlieb, S. M. Armour, M. H. Harris, and C. B. Thompson, "Mitochondrial membrane potential regulates matrix configuration and cytochrome c release during apoptosis," Cell Death and Differentiation, vol. 10, pp. 709-717, 2003, http://www.ncbi.nlm.nih.gov/pubmed/ 12761579.

[58] N. K. Mondal, E. N. Sorensen, E. D. Feller, S. M. Pham, B. P. Griffith, and Z. J. Wu, "Systemic inflammatory response syndrome after contentious-flow left ventricular assist device implantation and change in platelet mitochondrial membrane potential," Journal of Cardiac Failure, vol. 21, pp. 564-571, 2015, http://www.ncbi.nlm.nih.gov/pubmed/ 25921521.

[59] I. Kokkinopoulos, A. Colman, C. Hogg, J. Heckenlively, and G. Jeffery, "Age-related retinal inflammation is reduced by $670 \mathrm{~nm}$ light via increased mitochondrial membrane potential," Neurobiology of Aging, vol. 34, pp. 602-609, 2013, http://www.ncbi.nlm.nih.gov/pubmed/22595370.

[60] D. Bronner and M. O'Riordan, "Measurement of mitochondrial DNA release in response to er stress," Bio-Protocol, vol. 6, 2016http://www.ncbi.nlm.nih.gov/pubmed/31106234.

[61] N. Zamzami, P. Marchetti, M. Castedo et al., "Sequential reduction of mitochondrial transmembrane potential and generation of reactive oxygen species in early programmed cell death," Journal of Experimental Medicine, vol. 182, pp. 367-377, 1995, http://www.ncbi.nlm.nih.gov/pubmed/ 7629499.

[62] E. Gottlieb, M. G. Vander Heiden, and C. B. Thompson, "Bcl$\mathrm{x}(\mathrm{l})$ prevents the initial decrease in mitochondrial membrane potential and subsequent reactive oxygen species production during tumor necrosis factor alpha-induced apoptosis," Molecular and Cellular Biology, vol. 20, pp. 5680-5689, 2000, http://www.ncbi.nlm.nih.gov/pubmed/10891504.

[63] J. A. MacDonald, N. Kura, C. Sussman, and D. C. Woods, "Mitochondrial membrane depolarization enhances trailinduced cell death in adult human granulosa tumor cells, kgn, through inhibition of birc5," Journal of Ovarian Research, vol. 11, p. 89, 2018, http://www.ncbi.nlm.nih.gov/ pubmed/30326924.

[64] R. Rizzuto, P. Pinton, W. Carrington et al., "Close contacts with the endoplasmic reticulum as determinants of mitochondrial ca2+ responses," Science, vol. 280, no. 5370, pp. 1763-1766, 1998.

[65] A. R. Marks, "Intracellular calcium-release channels: regulators of cell life and death," American Journal of Physiology. Heart and Circulatory Physiology, vol. 272, pp. H597-H605, 1997, http://www.ncbi.nlm.nih.gov/pubmed/9124414.

[66] V. Granatiero, D. De Stefani, and R. Rizzuto, "Mitochondrial calcium handling in physiology and disease," Advances in Experimental Medicine and Biology, vol. 982, pp. 25-47, 2017, http://www.ncbi.nlm.nih.gov/pubmed/28551780.
[67] T. Finkel, S. Menazza, K. M. Holmström et al., "The ins and outs of mitochondrial calcium," Circulation Research, vol. 116, pp. 1810-1819, 2015, http://www.ncbi.nlm.nih .gov/pubmed/25999421.

[68] P. R. Territo, V. K. Mootha, S. A. French, and R. S. Balaban, " $\mathrm{Ca}(2+)$ activation of heart mitochondrial oxidative phosphorylation: role of the $\mathrm{f}(0) / \mathrm{f}(1)$-atpase," American Journal of Physiology-Cell Physiology, vol. 278, pp. C423-C435, 2000, http://www.ncbi.nlm.nih.gov/pubmed/10666039.

[69] B. Glancy, W. T. Willis, D. J. Chess, and R. S. Balaban, "Effect of calcium on the oxidative phosphorylation cascade in skeletal muscle mitochondria," Biochemistry, vol. 52, pp. 27932809, 2013, http://www.ncbi.nlm.nih.gov/pubmed/23547908.

[70] P. Bernardi, A. Rasola, M. Forte, and G. Lippe, “The mitochondrial permeability transition pore: channel formation by $\mathrm{f}$-atp synthase, integration in signal transduction, and role in pathophysiology," Physiological Reviews, vol. 95, pp. 11111155, 2015, http://www.ncbi.nlm.nih.gov/pubmed/ 26269524.

[71] S. Hurst, J. Hoek, and S. S. Sheu, "Mitochondrial $\mathrm{Ca}(2+)$ and regulation of the permeability transition pore," Journal of Bioenergetics and Biomembranes, vol. 49, pp. 27-47, 2017, http://www.ncbi.nlm.nih.gov/pubmed/27497945.

[72] T. Ren, H. Zhang, J. Wang et al., "MCU-dependent mitochondrial $\mathrm{Ca}^{2+}$ inhibits $\mathrm{NAD}^{+} / \mathrm{SIRT} 3 / \mathrm{SOD} 2$ pathway to promote ROS production and metastasis of HCC cells," Oncogene, vol. 36, no. 42, pp. 5897-5909, 2017.

[73] J. C. Liu, J. Liu, K. M. Holmström et al., "Micu1 serves as a molecular gatekeeper to prevent in vivo mitochondrial calcium overload," Cell Reports, vol. 16, no. 6, pp. 1561-1573, 2016.

[74] J. M. Baughman, F. Perocchi, H. S. Girgis et al., "Integrative genomics identifies mcu as an essential component of the mitochondrial calcium uniporter," Nature, vol. 476, no. 7360, pp. 341-345, 2011.

[75] D. de Stefani, R. Rizzuto, and T. Pozzan, "Enjoy the trip: calcium in mitochondria back and forth," Annual Review of Biochemistry, vol. 85, no. 1, pp. 161-192, 2016.

[76] Y. Tan, D. Mui, S. Toan, P. Zhu, R. Li, and H. Zhou, "Serca overexpression improves mitochondrial quality control and attenuates cardiac microvascular ischemia-reperfusion injury," Molecular Therapy-Nucleic Acids, vol. 22, pp. 696707, 2020.

[77] T. I. Peng and M. J. Jou, "Oxidative stress caused by mitochondrial calcium overload," Annals of the New York Academy of Sciences, vol. 1201, no. 1, pp. 183-188, 2010.

[78] L. Pan, B. J. Huang, X. E. Ma et al., "Mir-25 protects cardiomyocytes against oxidative damage by targeting the mitochondrial calcium uniporter," International Journal of Molecular Sciences, vol. 16, no. 12, pp. 5420-5433, 2015.

[79] E. Cadenas and A. Boveris, "Enhancement of hydrogen peroxide formation by protophores and ionophores in antimycin-supplemented mitochondria," The Biochemical Journal, vol. 188, no. 1, pp. 31-37, 1980.

[80] Y. Liao, Y. Hao, H. Chen, Q. He, Z. Yuan, and J. Cheng, "Mitochondrial calcium uniporter protein $\mathrm{mcu}$ is involved in oxidative stress-induced cell death," Protein \& Cell, vol. 6, no. 6, pp. 434-442, 2015.

[81] Y. Zu, L. J. Wan, S. Y. Cui, Y. P. Gong, and C. L. Li, “The mitochondrial $\mathrm{Na}(+) / \mathrm{Ca}(2+)$ exchanger may reduce high glucose-induced oxidative stress and nucleotide-binding 
oligomerization domain receptor 3 inflammasome activation in endothelial cells," Journal of Geriatric Cardiology, vol. 12, no. 3, pp. 270-278, 2015.

[82] A. A. Starkov, C. Chinopoulos, and G. Fiskum, "Mitochondrial calcium and oxidative stress as mediators of ischemic brain injury," Cell Calcium, vol. 36, no. 3-4, pp. 257-264, 2004.

[83] Q. Liu, D. Zhang, D. Hu, X. Zhou, and Y. Zhou, "The role of mitochondria in nlrp3 inflammasome activation," Molecular Immunology, vol. 103, pp. 115-124, 2018.

[84] K. Triantafilou, T. R. Hughes, M. Triantafilou, and B. P. Morgan, "The complement membrane attack complex triggers intracellular $\mathrm{Ca}^{2+}$ fluxes leading to nlrp3 inflammasome activation," Journal of Cell Science, vol. 126, pp. 2903-2913, 2013.

[85] A. Rimessi, C. Pozzato, L. Carparelli et al., "Pharmacological modulation of mitochondrial calcium uniporter controls lung inflammation in cystic fibrosis," Science Advances, vol. 6, no. 19, article eaax9093, 2020.

[86] A. Rimessi, V. Bezzerri, S. Patergnani, S. Marchi, G. Cabrini, and P. Pinton, "Mitochondrial $\mathrm{Ca}^{2+}$-dependent NLRP3 activation exacerbates the Pseudomonas aeruginosa-driven inflammatory response in cystic fibrosis," Nature Communications, vol. 6, no. 1, p. 6201, 2015.

[87] E. I. Elliott and F. S. Sutterwala, "Initiation and perpetuation of nlrp3 inflammasome activation and assembly," Immunological Reviews, vol. 265, no. 1, pp. 35-52, 2015.

[88] T. Horng, "Calcium signaling and mitochondrial destabilization in the triggering of the nlrp3 inflammasome," Trends in Immunology, vol. 35, no. 6, pp. 253-261, 2014.

[89] F. Correa, N. Pavón, M. Buelna-Chontal, N. Chiquete-Félix, L. Hernández-Esquivel, and E. Chávez, "Calcium induces mitochondrial oxidative stress because of its binding to adenine nucleotide translocase," Cell Biochemistry and Biophysics, vol. 76, no. 4, pp. 445-450, 2018.

[90] A. Görlach, K. Bertram, S. Hudecova, and O. Krizanova, "Calcium and ros: a mutual interplay," Redox Biology, vol. 6, pp. 260-271, 2015.

[91] M. Aldakkak, D. F. Stowe, R. K. Dash, and A. K. S. Camara, "Mitochondrial handling of excess $\mathrm{Ca}^{2+}$ is substratedependent with implications for reactive oxygen speciesgeneration," Free Radical Biology \& Medicine, vol. 56, pp. 193-203, 2013.

[92] S. Y. Jeong and D. W. Seol, "The role of mitochondria in apoptosis," BMB Reports, vol. 41, no. 1, pp. 11-22, 2008.

[93] N. Xie, C. Wu, C. Wang et al., "Inhibition of the mitochondrial calcium uniporter inhibits $\mathrm{A} \beta$-induced apoptosis by reducing reactive oxygen species-mediated endoplasmic reticulum stress in cultured microglia," Brain Research, vol. 1676, pp. 100-106, 2017.

[94] E. Basso, L. Fante, J. Fowlkes, V. Petronilli, M. A. Forte, and P. Bernardi, "Properties of the Permeability Transition Pore in Mitochondria Devoid of Cyclophilin D," The Journal of Biological Chemistry, vol. 280, no. 19, pp. 1855818561, 2005.

[95] G. Hajnóczky, G. Csordás, S. Das et al., "Mitochondrial calcium signalling and cell death: approaches for assessing the role of mitochondrial $\mathrm{Ca}^{2+}$ uptake in apoptosis," Cell Calcium, vol. 40, no. 5-6, pp. 553-560, 2006.

[96] S. C. Xu, Y. B. Chen, H. Lin et al., "Damage to mtdna in liver injury of patients with extrahepatic cholestasis: the protective effects of mitochondrial transcription factor a," Free Radical Biology \& Medicine, vol. 52, no. 9, pp. 1543-1551, 2012.

[97] X. W. Huang, Q. Zhao, D. Z. Chen, and L. S. Zhang, "Mutations in the d-loop region of mitochondrial DNA and the ros level in the tissue of hepatocellular carcinoma," Yi Chuan, vol. 27, no. 1, pp. 14-20, 2005.

[98] W. Bao, H. Xia, Y. Liang et al., "Toll-like receptor 9 can be activated by endogenous mitochondrial DNA to induce podocyte apoptosis," Scientific Reports, vol. 6, no. 1, p. 22579, 2016.

[99] A. Rodríguez-Nuevo, A. Díaz-Ramos, E. Noguera et al., "Mitochondrial DNA and tlr9 drive muscle inflammation upon opal deficiency," The EMBO Journal, vol. 37, no. 10, 2018.

[100] J. Bai, C. Cervantes, J. Liu et al., "Dsba-l prevents obesityinduced inflammation and insulin resistance by suppressing the mtdna release-activated cgas-cgamp-sting pathway," Proceedings of the National Academy of Sciences of the United States of America, vol. 114, no. 46, pp. 12196-12201, 2017.

[101] J. S. Riley, G. Quarato, C. Cloix et al., "Mitochondrial inner membrane permeabilisation enables mtdna release during apoptosis," The EMBO Journal, vol. 37, no. 17, 2018.

[102] H. Rizwan, S. Pal, S. Sabnam, and A. Pal, "High glucose augments ros generation regulates mitochondrial dysfunction and apoptosis via stress signalling cascades in keratinocytes," Life Sciences, vol. 241, p. 117148, 2020.

[103] Y. Yu, Y. Liu, W. An, J. Song, Y. Zhang, and X. Zhao, "Sting-mediated inflammation in kupffer cells contributes to progression of nonalcoholic steatohepatitis," The Journal of Clinical Investigation, vol. 129, no. 2, pp. 546-555, 2019.

[104] C. A. Pereira, D. Carlos, N. S. Ferreira et al., "Mitochondrial DNA promotes nlrp3 inflammasome activation and contributes to endothelial dysfunction and inflammation in type 1 diabetes," Frontiers in Physiology, vol. 10, p. 1557, 2019.

[105] K. Shimada, T. R. Crother, J. Karlin et al., "Oxidized mitochondrial DNA activates the nlrp3 inflammasome during apoptosis," Immunity, vol. 36, no. 3, pp. 401-414, 2012.

[106] J. H. Bae, S. I. I. Jo, S. J. Kim et al., "Circulating cell-free mtdna contributes to aim2 inflammasome-mediated chronic inflammation in patients with type 2 diabetes," Cell, vol. 8 , no. 4 , p. 328, 2019.

[107] L. Wang, M. Zheng, S. Zhang, C. Zhao, W. Kang, and $\mathrm{K}$. Wang, "Roles of mtDNA damage and disordered $\mathrm{Ca}^{2+}$ homeostasis in the joint toxicities of cadmium and BDE209," Ecotoxicology and Environmental Safety, vol. 186, p. 109767, 2019.

[108] Z. Ding, S. Liu, X. Wang et al., "Cross-talk between pcsk9 and damaged mtdna in vascular smooth muscle cells: role in apoptosis," Antioxidants \& Redox Signaling, vol. 25, no. 18, pp. 997-1008, 2016.

[109] A. Rongvaux, R. Jackson, C. C. Harman et al., "Apoptotic caspases prevent the induction of type i interferons by mitochondrial DNA," Cell, vol. 159, no. 7, pp. 1563-1577, 2014.

[110] I. Novak, "Mitophagy: a complex mechanism of mitochondrial removal," Antioxidants \& Redox Signaling, vol. 17, no. 5, pp. 794-802, 2012.

[111] S. Mehrabani, M. Bagherniya, G. Askari, M. I. Read, and A. Sahebkar, "The effect of fasting or calorie restriction on mitophagy induction: a literature review," Journal of Cachexia, Sarcopenia and Muscle, vol. 11, no. 6, pp. 1447$1458,2020$. 
[112] F. Koyano, K. Okatsu, H. Kosako et al., "Ubiquitin is phosphorylated by pink1 to activate parkin," Nature, vol. 510, no. 7503, pp. 162-166, 2014.

[113] M. Lazarou, D. A. Sliter, L. A. Kane et al., "The ubiquitin kinase pink1 recruits autophagy receptors to induce mitophagy," Nature, vol. 524, no. 7565, pp. 309-314, 2015.

[114] P. A. Ney, "Mitochondrial autophagy: origins, significance, and role of bnip3 and nix," Biochimica et Biophysica Acta, vol. 1853, no. 10, pp. 2775-2783, 2015.

[115] J. Wang, P. Zhu, R. Li, J. Ren, and H. Zhou, "Fundc1-dependent mitophagy is obligatory to ischemic preconditioningconferred renoprotection in ischemic aki via suppression of drp1-mediated mitochondrial fission," Redox Biology, vol. 30, p. 101415, 2020.

[116] H. Zhou, P. Zhu, J. Wang, H. Zhu, J. Ren, and Y. Chen, "Pathogenesis of cardiac ischemia reperfusion injury is associated with CK $2 \alpha$-disturbed mitochondrial homeostasis via suppression of FUNDC1-related mitophagy," Cell Death and Differentiation, vol. 25, no. 6, pp. 1080-1093, 2018.

[117] H. Zhou, P. Zhu, J. Wang, S. Toan, and J. Ren, "DNA-pkcs promotes alcohol-related liver disease by activating drp1related mitochondrial fission and repressing fundc1required mitophagy," Signal Transduction and Targeted Therapy, vol. 4, no. 1, p. 56, 2019.

[118] B. L. Baechler, D. Bloemberg, and J. Quadrilatero, “Mitophagy regulates mitochondrial network signaling, oxidative stress, and apoptosis during myoblast differentiation," Autophagy, vol. 15, no. 9, pp. 1606-1619, 2019.

[119] Y. Wang, J. Shen, Y. Chen et al., "Pink1 protects against oxidative stress induced senescence of human nucleus pulposus cells via regulating mitophagy," Biochemical and Biophysical Research Communications, vol. 504, no. 2, pp. 406-414, 2018.

[120] Z. Zhang, T. Zhang, R. Feng, H. Huang, T. Xia, and C. Sun, "Circarf3 alleviates mitophagy-mediated inflammation by targeting mir-103/traf3 in mouse adipose tissue," Molecular Therapy-Nucleic Acids, vol. 14, pp. 192-203, 2019.

[121] R. Sebori, A. Kuno, R. Hosoda, T. Hayashi, and Y. Horio, "Resveratrol decreases oxidative stress by restoring mitophagy and improves the pathophysiology of dystrophindeficient $\mathrm{mdx}$ mice," Oxidative Medicine and Cellular Longevity, vol. 2018, Article ID 9179270, 13 pages, 2018.

[122] T. Zhang, P. Wu, E. Budbazar et al., "Mitophagy reduces oxidative stress via keap1 (kelch-like epichlorohydrin-associated protein 1)/nrf2 (nuclear factor-e2-related factor 2)/phb2 (prohibitin 2) pathway after subarachnoid hemorrhage in rats," Stroke, vol. 50, no. 4, pp. 978-988, 2019.

[123] L. Kang, S. Liu, J. Li, Y. Tian, Y. Xue, and X. Liu, "Parkin and nrf2 prevent oxidative stress-induced apoptosis in intervertebral endplate chondrocytes via inducing mitophagy and antioxidant defenses," Life Sciences, vol. 243, p. 117244, 2020.

[124] D. R. Green, L. Galluzzi, and G. Kroemer, "Mitochondria and the autophagy-inflammation-cell death axis in organismal aging," Science, vol. 333, no. 6046, pp. 1109-1112, 2011.

[125] A. M. Pickrell, C. H. Huang, S. R. Kennedy et al., "Endogenous parkin preserves dopaminergic substantia nigral neurons following mitochondrial DNA mutagenic stress," Neuron, vol. 87, no. 2, pp. 371-381, 2015.

[126] A. Trifunovic, A. Wredenberg, M. Falkenberg et al., "Premature ageing in mice expressing defective mitochondrial DNA polymerase," Nature, vol. 429, no. 6990, pp. 417423, 2004.
[127] Q. Lin, S. Li, N. Jiang et al., "Pink1-parkin pathway of mitophagy protects against contrast-induced acute kidney injury via decreasing mitochondrial ros and nlrp3 inflammasome activation," Redox Biology, vol. 26, p. 101254, 2019.

[128] D. A. Sliter, J. Martinez, L. Hao et al., "Parkin and pink1 mitigate sting-induced inflammation," Nature, vol. 561, no. 7722, pp. 258-262, 2018.

[129] Z. Zhong, A. Umemura, E. Sanchez-Lopez et al., "NF- $\kappa$ B Restricts Inflammasome Activation via Elimination of Damaged Mitochondria," Cell, vol. 164, no. 5, pp. 896-910, 2016.

[130] Y. Wang, X. Mao, H. Chen et al., "Dexmedetomidine alleviates lps-induced apoptosis and inflammation in macrophages by eliminating damaged mitochondria via pink1 mediated mitophagy," International Immunopharmacology, vol. 73, pp. 471-481, 2019.

[131] R. Wei, J. Cao, and S. Yao, "Matrine promotes liver cancer cell apoptosis by inhibiting mitophagy and pink1/parkin pathways," Cell Stress \& Chaperones, vol. 23, no. 6, pp. 12951309, 2018.

[132] L. Si, J. Fu, W. Liu et al., "Silibinin-induced mitochondria fission leads to mitophagy, which attenuates silibinin-induced apoptosis in mcf-7 and mda-mb-231 cells," Archives of Biochemistry and Biophysics, vol. 685, p. 108284, 2020.

[133] A. Corlățeanu, I. Odajiu, V. Botnaru, and S. Cemirtan, "From smoking to copd-current approaches," Pneumologia, vol. 65, no. 1, pp. 20-23, 2016.

[134] A. T. Lo Tam Loi, S. J. M. Hoonhorst, L. Franciosi et al., "Acute and chronic inflammatory responses induced by smoking in individuals susceptible and non-susceptible to development of copd: from specific disease phenotyping towards novel therapy. Protocol of a cross-sectional study," BMJ Open, vol. 3, no. 2, p. e002178, 2013.

[135] R. F. Hoffmann, S. Zarrintan, S. M. Brandenburg et al., "Prolonged cigarette smoke exposure alters mitochondrial structure and function in airway epithelial cells," Respiratory Research, vol. 14, no. 1, p. 97, 2013.

[136] B. M. Fischer, J. Voynow, and A. Ghio, "Copd: balancing oxidants and antioxidants," International Journal of Chronic Obstructive Pulmonary Disease, vol. 10, pp. 261-276, 2015.

[137] on behalf of the COPDMAP consortium, G. Haji, C. H. Wiegman et al., "Mitochondrial dysfunction in airways and quadriceps muscle of patients with chronic obstructive pulmonary disease," Respiratory Research, vol. 21, no. 1, p. 262, 2020.

[138] C. H. Wiegman, C. Michaeloudes, G. Haji et al., "Oxidative stress-induced mitochondrial dysfunction drives inflammation and airway smooth muscle remodeling in patients with chronic obstructive pulmonary disease," The Journal of Allergy and Clinical Immunology, vol. 136, no. 3, pp. 769780, 2015.

[139] L. Puente-Maestu, A. Tejedor, A. Lázaro et al., "Site of mitochondrial reactive oxygen species production in skeletal muscle of chronic obstructive pulmonary disease and its relationship with exercise oxidative stress," American Journal of Respiratory Cell and Molecular Biology, vol. 47, no. 3, pp. 358-362, 2012.

[140] T. Ahmad, I. K. Sundar, C. A. Lerner et al., "Impaired mitophagy leads to cigarette smoke stress-induced cellular senescence: implications for chronic obstructive pulmonary disease," The FASEB Journal, vol. 29, no. 7, pp. 2912-2929, 2015. 
[141] S. Chen, Y. Wang, H. Zhang et al., "The antioxidant mitoq protects against cse-induced endothelial barrier injury and inflammation by inhibiting ros and autophagy in human umbilical vein endothelial cells," International Journal of Biological Sciences, vol. 15, no. 7, pp. 1440-1451, 2019.

[142] S. M. Cloonan, K. Glass, M. E. Laucho-Contreras et al., "Mitochondrial iron chelation ameliorates cigarette smokeinduced bronchitis and emphysema in mice," Nature Medicine, vol. 22, no. 2, pp. 163-174, 2016.

[143] K. Wu, G. Luan, Y. Xu et al., "Cigarette smoke extract increases mitochondrial membrane permeability through activation of adenine nucleotide translocator (ant) in lung epithelial cells," Biochemical and Biophysical Research Communications, vol. 525, no. 3, pp. 733-739, 2020.

[144] T. Zhou, Y. Hu, Y. Wang et al., "Fine particulate matter $\left(\mathrm{pm}_{2.5}\right)$ aggravates apoptosis of cigarette-inflamed bronchial epithelium in vivo and vitro," Environmental Pollution, vol. 248, pp. 1-9, 2019.

[145] J. Mao, Y. Li, S. Li et al., "Bufei jianpi granules reduce quadriceps muscular cell apoptosis by improving mitochondrial function in rats with chronic obstructive pulmonary disease," Evidence-based Complementary and Alternative Medicine, vol. 2019, Article ID 1216305, 9 pages, 2019.

[146] A. Petit, L. Knabe, K. Khelloufi et al., "Bronchial epithelial calcium metabolism impairment in smokers and chronic obstructive pulmonary disease. Decreased orai3 signaling," American Journal of Respiratory Cell and Molecular Biology, vol. 61, no. 4, pp. 501-511, 2019.

[147] S. Manral, S. Bhatia, R. Sinha et al., "Normalization of deranged signal transduction in lymphocytes of copd patients by the novel calcium channel blocker h-dhpm," Biochimie, vol. 93, no. 7, pp. 1146-1156, 2011.

[148] W. Z. Zhang, K. L. Hoffman, K. T. Schiffer et al., “Association of plasma mitochondrial DNA with copd severity and progression in the spiromics cohort," Respiratory Research, vol. 22 , no. 1, p. 126, 2021.

[149] W. Z. Zhang, M. C. Rice, K. L. Hoffman et al., "Association of urine mitochondrial DNA with clinical measures of copd in the spiromics cohort," JCI Insight, vol. 5, no. 3, 2020.

[150] G. E. Carpagnano, D. Lacedonia, M. Malerba et al., "Analysis of mitochondrial DNA alteration in new phenotype acos," BMC Pulmonary Medicine, vol. 16, no. 1, p. 31, 2016.

[151] S. D. Pouwels, G. J. Zijlstra, M. van der Toorn et al., "Cigarette smoke-induced necroptosis and damp release trigger neutrophilic airway inflammation in mice," American Journal of Physiology. Lung Cellular and Molecular Physiology, vol. 310, no. 4, pp. L377-L386, 2016.

[152] S. Ito, J. Araya, Y. Kurita et al., "Park2-mediated mitophagy is involved in regulation of hbec senescence in copd pathogenesis," Autophagy, vol. 11, no. 3, pp. 547-559, 2015.

[153] J. Araya, K. Tsubouchi, N. Sato et al., "Prkn-regulated mitophagy and cellular senescence during copd pathogenesis," Autophagy, vol. 15, no. 3, pp. 510-526, 2019.

[154] K. Mizumura, S. M. Cloonan, K. Nakahira et al., "Mitophagydependent necroptosis contributes to the pathogenesis of copd," The Journal of Clinical Investigation, vol. 124, no. 9, pp. 3987-4003, 2014.

[155] M. Zhang, R. Shi, Y. Zhang et al., "Nix/bnip3l-dependent mitophagy accounts for airway epithelial cell injury induced by cigarette smoke," Journal of Cellular Physiology, vol. 234, no. 8, pp. 14210-14220, 2019.
[156] W. Wen, G. Yu, W. Liu et al., "Silencing fundc1 alleviates chronic obstructive pulmonary disease by inhibiting mitochondrial autophagy and bronchial epithelium cell apoptosis under hypoxic environment," Journal of Cellular Biochemistry, vol. 120, no. 10, pp. 17602-17615, 2019.

[157] C. Veith, A. W. Boots, M. Idris, F. J. van Schooten, and A. van der Vliet, "Redox imbalance in idiopathic pulmonary fibrosis: a role for oxidant cross-talk between nadph oxidase enzymes and mitochondria," Antioxidants \& Redox Signaling, vol. 31, no. 14, pp. 1092-1115, 2019.

[158] M. Bueno, J. Calyeca, M. Rojas, and A. L. Mora, "Mitochondria dysfunction and metabolic reprogramming as drivers of idiopathic pulmonary fibrosis," Redox Biology, vol. 33, p. 101509, 2020.

[159] M. Bueno, Y. C. Lai, Y. Romero et al., "Pink1 deficiency impairs mitochondrial homeostasis and promotes lung fibrosis," The Journal of Clinical Investigation, vol. 125, no. 2, pp. 521-538, 2015.

[160] M. Selman, M. Rojas, A. Mora, and A. Pardo, "Aging and interstitial lung diseases: unraveling an old forgotten player in the pathogenesis of lung fibrosis," Seminars in Respiratory and Critical Care Medicine, vol. 31, no. 5, pp. 607-617, 2010.

[161] B. Ley, H. R. Collard, and T. E. King Jr., "Clinical course and prediction of survival in idiopathic pulmonary fibrosis," American Journal of Respiratory and Critical Care Medicine, vol. 183, no. 4, pp. 431-440, 2011.

[162] D. Álvarez, N. Cárdenes, J. Sellarés et al., "Ipf lung fibroblasts have a senescent phenotype," American Journal of Physiology. Lung Cellular and Molecular Physiology, vol. 313, no. 6, pp. L1164-L1173, 2017.

[163] M. Schuliga, D. V. Pechkovsky, J. Read et al., "Mitochondrial dysfunction contributes to the senescent phenotype of ipf lung fibroblasts," Journal of Cellular and Molecular Medicine, vol. 22, no. 12, pp. 5847-5861, 2018.

[164] J. L. Larson-Casey, J. S. Deshane, A. J. Ryan, V. J. Thannickal, and A. B. Carter, "Macrophage akt1 kinase-mediated mitophagy modulates apoptosis resistance and pulmonary fibrosis," Immunity, vol. 44, no. 3, pp. 582-596, 2016.

[165] S. J. Kim, P. Cheresh, R. P. Jablonski et al., "Mitochondrial catalase overexpressed transgenic mice are protected against lung fibrosis in part via preventing alveolar epithelial cell mitochondrial DNA damage," Free Radical Biology \& Medicine, vol. 101, pp. 482-490, 2016.

[166] V. Suryadevara, L. Huang, S. J. Kim et al., "Role of phospholipase $\mathrm{d}$ in bleomycin-induced mitochondrial reactive oxygen species generation, mitochondrial DNA damage, and pulmonary fibrosis," American Journal of Physiology. Lung Cellular and Molecular Physiology, vol. 317, no. 2, pp. L175-L187, 2019.

[167] S. J. Kim, P. Cheresh, R. Jablonski, D. Williams, and D. Kamp, "The role of mitochondrial DNA in mediating alveolar epithelial cell apoptosis and pulmonary fibrosis," International Journal of Molecular Sciences, vol. 16, no. 9, pp. 2148621519, 2015.

[168] I. Ganzleben, G. W. He, C. Günther et al., "Pgam5 is a key driver of mitochondrial dysfunction in experimental lung fibrosis," Cellular and Molecular Life Sciences, vol. 76, no. 23, pp. 4783-4794, 2019.

[169] K. Liu, Z. Zhan, W. Gao, J. Feng, and X. Xie, "Cyclosporine attenuates paraquat-induced mitophagy and pulmonary fibrosis," Immunopharmacology and Immunotoxicology, vol. 42, no. 2, pp. 138-146, 2020. 
[170] L. Gu, J. L. Larson-Casey, and A. B. Carter, "Macrophages utilize the mitochondrial calcium uniporter for profibrotic polarization," The FASEB Journal, vol. 31, no. 7, pp. 30723083, 2017.

[171] L. Gu, J. L. Larson Casey, S. A. Andrabi et al., "Mitochondrial calcium uniporter regulates PGC- $1 \alpha$ expression to mediate metabolic reprogramming in pulmonary fibrosis," Redox Biology, vol. 26, p. 101307, 2019.

[172] C. Ryu, H. Sun, M. Gulati et al., "Extracellular mitochondrial DNA is generated by fibroblasts and predicts death in idiopathic pulmonary fibrosis," American Journal of Respiratory and Critical Care Medicine, vol. 196, no. 12, pp. 1571-1581, 2017.

[173] S. M. Cloonan, "Circulating mitochondrial DNA as a mechanism-based, prognostic biomarker for idiopathic pulmonary fibrosis," American Journal of Respiratory and Critical Care Medicine, vol. 196, no. 12, pp. 1502-1504, 2017.

[174] R. P. Jablonski, S. J. Kim, P. Cheresh et al., "Sirt3 deficiency promotes lung fibrosis by augmenting alveolar epithelial cell mitochondrial DNA damage and apoptosis," The FASEB Journal, vol. 31, no. 6, pp. 2520-2532, 2017.

[175] S. Bindu, V. B. Pillai, A. Kanwal et al., "Sirt3 blocks myofibroblast differentiation and pulmonary fibrosis by preventing mitochondrial DNA damage," American Journal of Physiology. Lung Cellular and Molecular Physiology, vol. 312, no. 1, pp. L68-L78, 2017.

[176] K. Kobayashi, J. Araya, S. Minagawa et al., "Involvement of park2-mediated mitophagy in idiopathic pulmonary fibrosis pathogenesis," Journal of Immunology, vol. 197, no. 2, pp. 504-516, 2016.

[177] Y. Kurita, J. Araya, S. Minagawa et al., "Pirfenidone inhibits myofibroblast differentiation and lung fibrosis development during insufficient mitophagy," Respiratory Research, vol. 18, no. 1, p. 114, 2017.

[178] G. Yu, A. Tzouvelekis, R. Wang et al., "Thyroid hormone inhibits lung fibrosis in mice by improving epithelial mitochondrial function," Nature Medicine, vol. 24, no. 1, pp. 3949, 2018.

[179] C. D. Kidd, P. J. Thompson, L. Barrett, and S. Baltic, "Histone modifications and asthma. The interface of the epigenetic and genetic landscapes," American Journal of Respiratory Cell and Molecular Biology, vol. 54, no. 1, pp. 3-12, 2016.

[180] A. P. Baptist and P. J. Busse, "Asthma over the age of 65: all's well that ends well," The Journal of Allergy and Clinical Immunology. In Practice, vol. 6, no. 3, pp. 764-773, 2018.

[181] P. H. Reddy, "Mitochondrial dysfunction and oxidative stress in asthma: implications for mitochondria-targeted antioxidant therapeutics," Pharmaceuticals (Basel), vol. 4, no. 3, pp. 429-456, 2011.

[182] P. O. Girodet, B. Allard, M. Thumerel et al., "Bronchial smooth muscle remodeling in nonsevere asthma," American Journal of Respiratory and Critical Care Medicine, vol. 193, no. 6, pp. 627-633, 2016.

[183] T. Mitsui, H. Azuma, M. Nagasawa et al., "Chronic corticosteroid administration causes mitochondrial dysfunction in skeletal muscle," Journal of Neurology, vol. 249, no. 8, pp. 1004-1009, 2002.

[184] T. Mitsui, Y. Umaki, M. Nagasawa et al., "Mitochondrial damage in patients with long-term corticosteroid therapy: development of oculoskeletal symptoms similar to mitochon- drial disease," Acta Neuropathologica, vol. 104, no. 3, pp. 260-266, 2002.

[185] K. P. Hough, J. L. Trevor, J. G. Strenkowski et al., "Exosomal transfer of mitochondria from airway myeloid-derived regulatory cells to t cells," Redox Biology, vol. 18, pp. 54-64, 2018.

[186] Ederlé, Charles, Khayath et al., "Mitochondrial function in peripheral blood mononuclear cells (pbmc) is enhanced, together with increased reactive oxygen species, in severe asthmatic patients in exacerbation," Journal of Clinical Medicine, vol. 8, no. 10, p. 1613, 2019.

[187] O. A. Jaffer, A. B. Carter, P. N. Sanders et al., "Mitochondrialtargeted antioxidant therapy decreases transforming growth Factor- $\beta$-Mediated collagen production in a murine asthma model," American Journal of Respiratory Cell and Molecular Biology, vol. 52, no. 1, pp. 106-115, 2015.

[188] A. Theiler, T. Bärnthaler, W. Platzer et al., "Butyrate ameliorates allergic airway inflammation by limiting eosinophil trafficking and survival," The Journal of Allergy and Clinical Immunology, vol. 144, no. 3, pp. 764-776, 2019.

[189] H. Liu, S. Tao, H. Ma et al., "Functional changes of airway epithelial cells and mitochondria in rat models of asthenic lung and phlegm blocking combined with cough variant asthma," Experimental and Therapeutic Medicine, vol. 16, no. 6, pp. 5021-5024, 2018.

[190] R. K. Ramakrishnan, K. Bajbouj, M. Y. Hachim et al., "Enhanced mitophagy in bronchial fibroblasts from severe asthmatic patients," PLoS One, vol. 15, no. 11, article e0242695, 2020.

[191] T. Trian, G. Benard, H. Begueret et al., "Bronchial smooth muscle remodeling involves calcium-dependent enhanced mitochondrial biogenesis in asthma," The Journal of Experimental Medicine, vol. 204, no. 13, pp. 3173-3181, 2007.

[192] P. O. Girodet, G. Dournes, M. Thumerel et al., "Calcium channel blocker reduces airway remodeling in severe asthma. A proof-of-concept study," American Journal of Respiratory and Critical Care Medicine, vol. 191, no. 8, pp. 876-883, 2015.

[193] S. C. Sebag, O. M. Koval, J. D. Paschke, C. J. Winters, A. P. Comellas, and I. M. Grumbach, "Inhibition of the mitochondrial calcium uniporter prevents IL-13 and allergen- mediated airway epithelial apoptosis and loss of barrier function," Experimental Cell Research, vol. 362, no. 2, pp. 400-411, 2018.

[194] Y. Zhang, D. C. Do, X. Hu et al., "Camkii oxidation regulates cockroach allergen-induced mitophagy in asthma," The Journal of Allergy and Clinical Immunology, vol. 147, no. 4, pp. 1464-1477.e11, 2020.

[195] A. Flaquer, A. Heinzmann, S. Rospleszcz et al., "Association study of mitochondrial genetic polymorphisms in asthmatic children," Mitochondrion, vol. 14, no. 1, pp. 49-53, 2014.

[196] G. E. Carpagnano, G. Scioscia, D. Lacedonia et al., "Busqueda de marcadores de estres inflamatorio y oxidativo capaces de agrupar asma grave," Archivos de Bronconeumología, vol. 57 , no. 5 , pp. 338-344, 2020.

[197] K. G. Dimasuay, N. Schaunaman, R. J. Martin et al., "Parkin, an e3 ubiquitin ligase, enhances airway mitochondrial DNA release and inflammation," Thorax, vol. 75, no. 9, pp. 717724, 2020.

[198] Y. Han, L. Chen, H. Liu et al., "Airway epithelial cgas is critical for induction of experimental allergic airway inflammation," Journal of Immunology, vol. 204, no. 6, pp. 1437-1447, 2020. 
[199] S. Pan, S. D. Shah, R. A. Panettieri Jr., and D. A. Deshpande, "Bnip3 regulates airway smooth muscle cell focal adhesion and proliferation," American Journal of Physiology. Lung Cellular and Molecular Physiology, vol. 317, no. 6, pp. L758L767, 2019.

[200] A. Chaudhry, R. Shi, and D. S. Luciani, "A pipeline for multidimensional confocal analysis of mitochondrial morphology, function, and dynamics in pancreatic $\beta$-cells," American Journal of Physiology. Endocrinology and Metabolism, vol. 318, no. 2, pp. E87-E101, 2020.

[201] L. S. Ludwig, C. A. Lareau, J. C. Ulirsch et al., "Lineage tracing in humans enabled by mitochondrial mutations and singlecell genomics," Cell, vol. 176, no. 6, pp. 1325-1339.e22, 2019. 